\title{
CARACTERIZACIÓN DE LOS CAMBIOS EN LA DESIGUALDAD EN COLOMBIA, MEDIANTE DESCOMPOSICIONES (1992-2012)
}

\author{
Cristian Felipe Orjuela ${ }^{1}$ \\ Tesis \\ Maestría en Economía \\ Universidad Nacional de La Plata
}

Director

Pablo Gluzmann

[Fecha: 17/10/2014]

Clasificación JEL: C15, D31, I21, J23, J31

\footnotetext{
${ }^{1}$ Universidad Nacional de La Plata. Email: <cristian.orjuela10@gmail.com>
} 


\section{RESUMEN}

Este documento intenta analizar la evolución de la desigualdad en ingresos y sus determinantes para Colombia durante el período 1992-2012. Para ello se implementan dos metodologías complementarias, por un lado, se aplica una estrategia de descomposiciones microeconométricas implementada por Gasparini et al. (2005). Dicho enfoque metodológico permite evaluar el efecto de algunos factores que se creen explican los cambios distributivos en Colombia durante el período 1992-2012, en cuanto a dirección y magnitud. Luego se realiza un ejercicio complementario mediante la descomposición del índice de Gini por fuente de ingresos basada en Lerman y Yitzhaki (1985), para evaluar la tendencia y la contribución de cada una de las fuentes de ingreso en la desigualdad para el período bajo análisis.

Los resultados de la primera metodología muestran que los factores más importantes en explicar los cambios en la distribución del ingreso para la década de los noventa fueron los retornos a la educación, seguido de los factores no observados y la estructura educativa. Para la década siguiente, tenemos dos conjuntos claros de factores que van en direcciones opuestas. Por un lado, tenemos el conjunto de los retornos a las características observables, los factores no observados y la estructura educativa, que en mayor o menor medida tienen un efecto igualador durante toda la década. Por otro lado, tenemos los efectos de horas de trabajo y la estructura del empleo que presentan un efecto desigualador durante ese período.

El análisis de descomposición por fuente de ingreso muestra que los ingresos laborales son los más importantes en determinar la desigualdad durante las últimas dos décadas, aunque pierden relevancia y los ingresos no laborales aumentan su importancia. La renta imputada presenta diferencias importantes en términos de nivel cuando se utilizan metodologías alternativas, pero la evolución es similar para dichas metodologías de imputación. 


\section{INTRODUCCIÓN}

Colombia es un país que ha atravesado por grandes cambios en la distribución del ingreso en las dos últimas décadas. Según las encuestas de hogares, se observa un incremento importante en la concentración del ingreso urbano, medida con el índice de Gini. Para la década de los noventa, el Gini del ingreso per cápita familiar pasa del 47.5 en 1992 al 54.7 en 2000. Para la década siguiente la desigualdad no presenta un patrón claro; la desigualdad cae entre 2002 y 2003, y luego presenta cambios pequeños, primero crecientes y luego decrecientes hasta 2010, con niveles promedio de 55.6 entre 2002 y 2010 y una reducción de 0.97 puntos. Sin embargo, durante los últimos dos años se presenta una importante reducción de 2.1 puntos hasta llegar a un nivel del índice de Gini de 53.5 en 2012 (ver gráfico 1.1), por lo cual es relevante preguntarse por los factores subyacentes a esas dinámicas durante estas dos décadas en la distribución del ingreso.

\section{Gráfico 1.1. Coeficiente de Gini del ingreso per cápita de Colombia, 1992-2012}

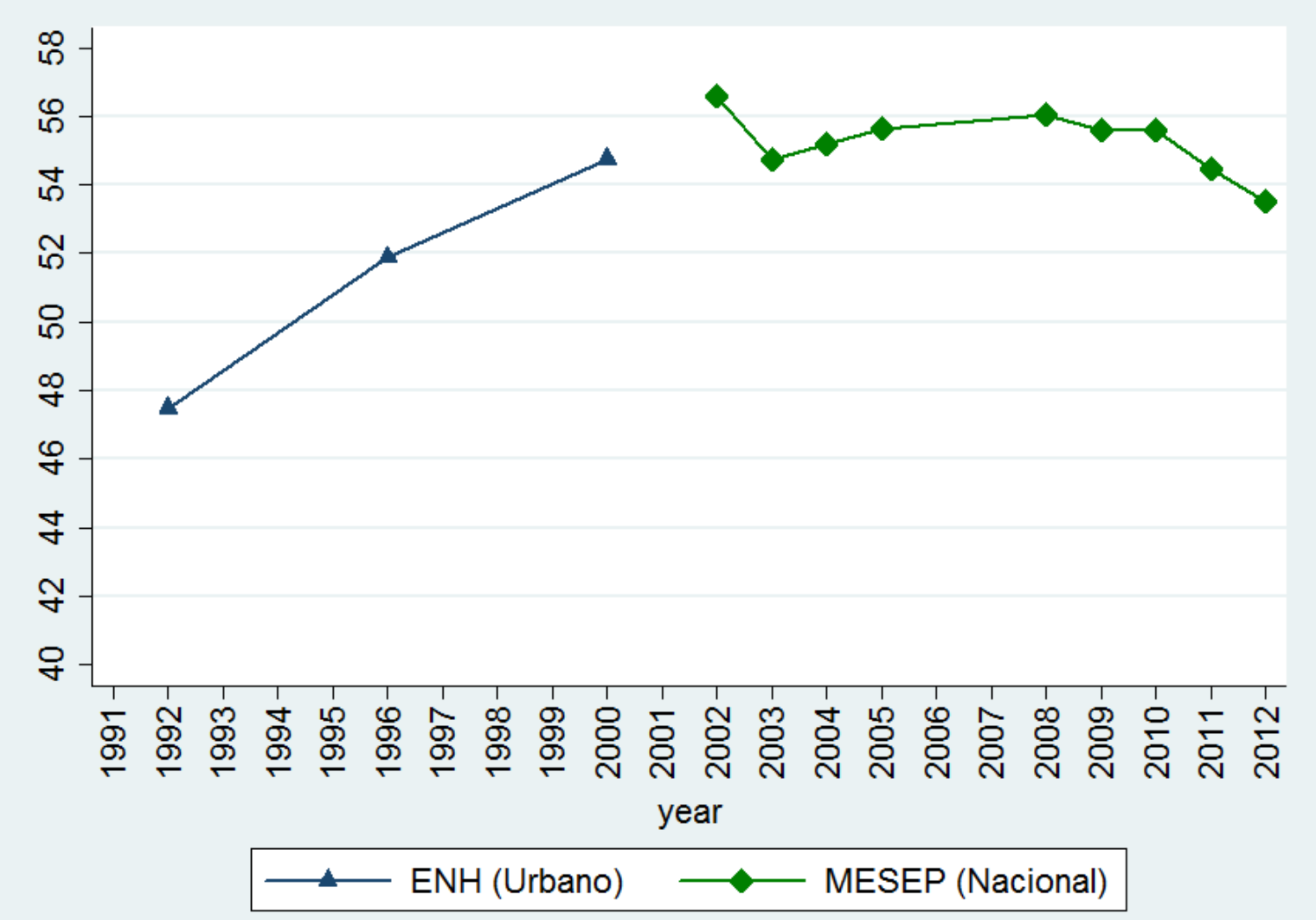

Fuente: Cálculos propios basados en la ENH y MESEP. 
Las razones que explican estos cambios en la desigualdad son complejas, dado que son el resultado de muchos factores que pueden determinar los cambios distributivos en las últimas dos décadas. Algunos de los trabajos sobre los determinantes de la desigualdad en Colombia afirman que el sostenido incremento de la desigualdad durante la segunda mitad de los noventa se explica, por un lado, por las reformas estructurales a inicios de esa década (principalmente la liberación comercial y de los mercados laborales). ${ }^{2}$ Por otro lado, unos trabajos se concentran en los cambios en la estructura educativa y los retornos al capital humano. ${ }^{3}$ En la década de los 2000 se observa una tendencia hacia la convergencia de los salarios por niveles educativos, pero un incremento de la dispersión dentro de los niveles superiores. ${ }^{4}$

El presente estudio tiene como objetivo principal caracterizar los cambios de la desigualdad en Colombia para el período 1992-2012. Para ello se implementa la estrategia de descomposiciones microeconométricas aplicada por Gasparini et al. (2005) para el caso argentino. Esta estrategia es utilizada con el objeto de evaluar la magnitud y dirección de efectos que provocan los principales determinantes de los ingresos laborales sobre los cambios en el coeficiente de Gini del ingreso per cápita familiar. Adicionalmente se descompone del índice de Gini por fuente de ingresos basada en propuesta de Lerman y Yitzhaki (1985), para evaluar la tendencia y la contribución de cada una de las fuentes de ingreso en la desigualdad para el período bajo análisis.

Existen algunos trabajos sobre los determinantes de la desigualdad en Colombia que usan algunas de las técnicas de descomposiciones, ${ }^{5}$ con el análisis detallado de estos factores se hacen aportes interesantes para el diseño e implementación de políticas pertinentes para tratar de solucionar el problema socioeconómico de la inequidad en la distribución del ingreso.

La primera estrategia intenta ser un aporte sobre esa línea de investigación, cuya contribución es la aplicación de una metodología que hasta el momento no ha sido

\footnotetext{
${ }^{2}$ Bernal et al. (1997) exploran las relaciones entre condiciones macroeconómicas y urbanas de la distribución del ingreso. Bernal y Cárdenas (1999) profundizan en los efectos de las reformas estructurales, principalmente la liberación comercial.

${ }^{3}$ Un conjunto importante de trabajos sigue la hipótesis del cambio técnico sesgado hacia trabajo calificado y encuentra evidencia a favor de ésta (ver la sección 2).

${ }^{4}$ Ver Vargas, C. (2011) y Posso, C. (2008).

${ }^{5}$ La siguiente sección se comenta los principales.
} 
implementada al caso colombiano. Los resultados obtenidos pueden ser útiles para hacer comparaciones con otros países latinoamericanos, dado que trabajos como los realizados por Gasparini et al. (2005), Haimovich et al. (2006), Zoloa (2011), entre otros, son contribuciones a la búsqueda de explicaciones a los cambios en la desigualdad de ingresos que usan la misma metodología. La segunda estrategia es complementaria de la primera, en el sentido de que permite analizar la importancia relativa de fuentes de ingreso distintas a las laborales en la desigualdad (dado que es dificultoso establecer sus determinantes). Pero adicionalmente, dadas las limitaciones en términos de comparabilidad de las encuestas en Colombia, el ejercicio permite analizar los efectos de metodologías alternativas para la comparación sobre las variables de interés.

En la siguiente sección se presenta una breve revisión de la literatura más relevante para el caso colombiano. Luego se da una breve descripción de las fuentes de información utilizadas en la sección tres. La sección cuatro analiza los determinantes de los ingresos laborales para para Colombia y su posible impacto sobre la desigualdad. La sección cinco implementa la estrategia de descomposiciones micro-econométricas. En la sección seis se realiza el ejercicio de descomposición por fuentes de ingreso; y finalmente, la sección siete contiene los comentarios finales.

\section{REVISIÓN DE LA LITERATURA}

Para el caso colombiano, los trabajos empíricos sobre la desigualdad del ingreso se pueden dividir en dos líneas importantes. Un primer grupo sigue la hipótesis de un cambio técnico sesgado hacia el trabajo calificado. Esta teoría establece que los desplazamientos en la demanda relativa de trabajo calificado con respecto al no calificado son mayores a los desplazamientos de la oferta relativa de trabajo, lo cual incrementa el diferencial de los salarios entre calificados y no calificados, siendo la explicación a los cambios en la desigualdad salarial. La segunda línea o grupo de trabajos exploran hipótesis alternativas y complementarias a la del cambio tecnológico sesgado. Estos trabajos analizan factores como las reformas estructurales, la reforma comercial de principios de los noventa, el desempleo, la informalidad, etc.

Dentro de la primera línea de trabajos, Núñez y Sánchez (1998) estudian para el período 1976-1997, la contribución de un conjunto de factores en la desigualdad de los salarios laborales urbanos, y el aporte de cada uno de esos factores al cambio en la 
desigualdad, medida como la varianza del logaritmo de los ingresos laborales y el índice de Gini. Mediante la metodología de Shorrocks (1982) se descompone la varianza del logaritmo de los ingresos laborales por componentes factoriales, para medir la contribución de cada uno de los factores y la contribución de cada uno al cambio en la distribución de ingresos laborales. El estudio concluye que el principal factor que contribuye a la desigualdad es la educación, debido a que la mayor rentabilidad de la educación superior tiene como consecuencia una mayor concentración de los ingresos laborales. El deterioro de la distribución también se explica por las diferencias de ingreso interregionales, el aumento del subempleo y la informalidad. Esta evidencia valida la hipótesis de que la mayor dispersión entre calificados y no calificados es determinada por el incremento de la demanda relativa por trabajadores calificados, a pesar de los incrementos en la oferta de trabajo calificado.

Bernal y Cárdenas (1999) analizan los cambios en la distribución del ingreso en Colombia entre 1976-1996 usando datos para las siete áreas metropolitanas en el sector manufacturero. La hipótesis principal es que las reformas estructurales de inicios de los noventa han estado relacionadas con una mayor concentración del ingreso en Colombia. Dentro del paquete de reformas, principalmente la liberación comercial no derivó en una reasignación entre sectores intensivos en trabajo calificado relativo a sectores intensivos en trabajo no calificado; por el contrario, esto favoreció la inversión en tecnologías complementarias a trabajadores calificados para todos los sectores de la producción. Para analizar los cambios en la demanda relativa de trabajo calificado con respecto al trabajo no calificado, los autores usan la metodología sugerida por Autor et al. (1998) que supone una función de oferta relativa inelástica en el corto plazo y una función de demanda de pendiente negativa. ${ }^{6}$ Los resultados sugieren que el cambio técnico complementario a trabajadores calificados ha sido el principal factor detrás de los cambios en la demanda relativa de trabajo.

Arango et al. (2004) analizan la evolución de los salarios reales urbanos de los ocupados tiempo completo entre 1984 y 2000. En una primera aproximación al entendimiento del aumento de la concentración de los ingresos salariales, se hace una

\footnotetext{
${ }^{6}$ Esta metodología usa una tecnología CES con dos factores, trabajo calificado y no calificado, para el diseño de inferencias acerca de la tasa de crecimiento de la demanda relativa de trabajo y oferta de trabajo calificado.
} 
descomposición del índice de Gini mediante la aplicación de la técnica de la varianza del logaritmo de Shorrocks (1982). Luego complementan el análisis siguiendo la línea de Katz y Murphy (1992), que consiste en suponer distintos valores para la elasticidad de sustitución entre el trabajo calificado y el no calificado, en una función de producción compatible con el cambio tecnológico sesgado. Los autores encuentran que la descomposición del índice de Gini muestra que la mayor desigualdad de los salarios provino de hombres y mujeres con alto nivel educativo, vinculados al sector privado. Los resultados también sugieren que hubo un cambio técnico sesgado a favor del trabajo calificado durante la década de los noventa. Posteriormente Vargas (2011), siguiendo la hipótesis y el diseño metodológico propuesto por Arango et al. (2004), amplía el período de análisis para Colombia desde 1984 hasta 2010 y encuentra evidencia a favor de la hipótesis del cambio técnico sesgado para Colombia en la década de los noventa, pero señala que ese efecto se ha desacelerado durante la década del 2000.

En el análisis descriptivo de las variables provenientes de Encuestas de Hogares, realizado por Bernal y Cárdenas (1999), se encuentra que éstas representaron el 60\% de la dispersión del ingreso en 1988 y el 70\% en 1996, sugiriendo que el resto de la desigualdad puede ser explicada por otros factores diferentes como las variables macroeconómicas. Esto da lugar al estudio de hipótesis alternativas que complementan la hipótesis cambio técnico sesgado. Por un lado tenemos a Bernal et al. (1997) quienes encuentran que el desempleo y la inflación tienen un efecto regresivo y significativo en la distribución del ingreso; sin embargo, el crecimiento del producto manufacturero y una moneda más depreciada parecen tener efectos progresivos en reducir la concentración del ingreso.

Siguiendo esta segunda línea de trabajos, Attanasio et al. (2004) profundizan en la relación entre la drástica disminución arancelaria desde la segunda mitad de los ochenta e inicios de los noventa con la distribución de salarios, identificando tres canales principales a través de los cuales afectó la distribución: (1) el incremento en las primas salariales fue debido principalmente al cambio técnico sesgado; sin embargo, los sectores con mayores reducciones arancelarias observaron un mayor incremento en las participaciones de trabajadores calificados; (2) las primas industriales también disminuyen fuertemente en aquellos sectores con mayores recortes arancelarios; y (3) los incrementos en la informalidad están relacionados con mayores recortes arancelarios y mayor exposición comercial. 
Entre los estudios recientes sobre la desigualdad del ingreso en Colombia se pueden destacar los trabajos de Tribin (2005) y Posso (2008). Mediante la técnica de Juhn Murphy y Prierce (1993), Tribín (2005) descompone los cambios en la desigualdad salarial de Bogotá en las características observables (educación y experiencia), cambios en los retornos de las características observables y cambios en los residuos, para el período comprendido entre 1976 y 2000. Los resultados muestran que el incremento de la desigualdad para el sub-período 1987 y 1997 fue determinado principalmente por los cambios en los retornos a la educación y los cambios en los residuos. Se concluye que los desplazamientos en la demanda relativa de trabajo mayores a los incrementos en la oferta relativa son los que explican el incremento de los retornos a la educación y, por lo tanto, el incremento en la desigualdad salarial. La importancia del componente residual puede deberse a un incremento de la valoración de las características no observables (precios relativos de dichas características).

El trabajo de Posso (2008) también estudia las desigualdades salariales pero mediante un ejercicio de descomposición de las diferencias salariales por percentiles, usando la metodología de Melly (2005), para el período 1985-2008 en Colombia. En este ejercicio descompone los cambios en la distribución de salarios en tres componentes: cambios en la distribución de las características, cambios en los coeficientes y cambios en los residuos. Como resultado obtiene que la evolución de la desigualdad salarial se encuentra explicada principalmente por aquellos asalariados con educación post-secundaria, los cuales reportan los mayores incrementos en retornos a la educación. Igualmente, existe una gran dispersión del efecto parámetros para el grupo con característica postsecundaria, a lo cual el autor infiere que la causa son diferencias en la calidad de las instituciones de educación postsecundaria.

A continuación se realiza una breve descripción de las fuentes de datos utilizadas en este estudio.

\section{DATOS UTILIZADOS}

Para la elaboración de este estudio se utilizó como fuente de datos la Encuesta Nacional de Hogares (ENH) para los años 1992 y 1996, y las encuestas empalmadas con la 
metodología del programa MESEP-DANE ${ }^{7}$ (MESEP en adelante) para el período 20022012. Es importante señalar que el período bajo análisis está sujeto a restricciones de comparabilidad debido a los cambios metodológicos de las encuestas de hogares de Colombia.

La ENH 1992 cubre sólo a los hogares urbanos, mientras que el resto de las encuestas incluyen hogares tanto urbanos como rurales, pero en la ENH 1996 la muestra de hogares rurales difiere en términos relativos en comparación con las encuestas de la siguiente década. Las encuestas de Colombia sufren importantes modificaciones a partir del año 2000, a nivel de diseño muestral, conceptual y de coberturas. Entre los principales cambios se pasó de una frecuencia de recolección trimestral o transversal a un sistema continuo de recolección bajo la denominación de Encuesta Continua de Hogares, se actualizó la distribución de espacial de la población y se realizaron cambios conceptuales en las definiciones de la fuerza de trabajo.

La MESEP es una metodología de empalme de las series de empleo, pobreza y desigualdad, debida a los cambios metodológicos entre la Encuesta Continua de Hogares y la Gran Encuesta Integrada de Hogares. ${ }^{8}$ Además, la metodología corrige algunos problemas relacionados con la construcción de la variable ingreso como la corrección por omisión, valores extremos, falsos ceros, entre otros.

Para la construcción de las variables de interés en el caso de las ENH se siguieron los lineamientos generales para la homogenización de los datos y construcción de las variables de ingreso; sin embargo, existen fuertes limitaciones de comparabilidad de éstas con las bases de datos MESEP. ${ }^{9}$ En este sentido, los resultados del presente trabajo deben analizarse con cuidado, debido a que las bases de datos de MESEP no son comparables con la metodología de la Encuesta Nacional de Hogares de los años noventa.

\footnotetext{
${ }^{7}$ Misión para el Empalme de las Series de Empleo, Pobreza y Desigualdad del Departamento Administrativo Nacional de Estadística

${ }^{8}$ La Encuesta Continua de Hogares cubre el período 2000-2005 y la Gran Encuesta Integrada de Hogares inicia desde el 2008.

${ }^{9}$ Las fuertes limitaciones entre ENH y MESEP, consisten en que, además de los cambios en el diseño muestral mencionados anteriormente, Mesep es también una metodología de armonización con la cual se corrigen varios problemas típicos de las encuestas de hogares, mediante técnicas estadísticas avanzadas y, que no se aplicaron a las bases ENH. Mientras que para la armonización de la ENH se realizó mediante lineamientos generales para homogenización de datos, estos no ser realizaron al nivel técnico de Mesep, dado que excedía los alcances del presente trabajo.
} 
Adicionalmente, pese a la metodología esfuerzo de homogeneización de MESEP, entre las encuestas ECH (2002-2005) y GEIH (2008-2012) existen limitaciones de comparabilidad, las principales diferencias son: la ampliación del tamaño de la muestra mensual, la introducción de nuevas preguntas y opciones de respuesta en el formulario, la actualización del marco muestral en todo el dominio territorial con base en el Censo 2005, e innovaciones de tipo tecnológico en la recolección de los datos. A su vez es importante resaltar que la metodología de empalme de MESEP se realizó sobre los ingresos agregados lo que limita de alguna forma la comparabilidad de la ECH (2002-2005) con la GEIH (2008-2012) al estudiar sus componentes desagregados. ${ }^{10}$

Dadas estas limitaciones, las dos décadas que abarca el estudio se dividen principalmente en tres sub-períodos: 1992-1996, 2002-2005 y 2005-2012. Las comparaciones del período 1996-2002 se realizan solo a título ilustrativo. La selección de estos años se caracteriza por presentar cierta estabilidad macroeconómica. La tabla 3.1 presenta una breve descripción de las principales encuestas utilizadas donde se evidencian algunos de los cambios en términos muestrales y de la población representada mencionados anteriormente. Adicionalmente, la tabla A.1 del Anexo muestra las variables usadas a lo largo del presente trabajo.

Tabla 3.1. Encuesta de hogares. Principales características

\begin{tabular}{|c|c|c|c|c|c|c|c|c|}
\hline \multirow{3}{*}{ Encuesta } & \multirow{3}{*}{ Año } & \multirow{3}{*}{ Período } & \multicolumn{4}{|c|}{ Observaciones } & \multicolumn{2}{|c|}{ Porcentaje Urbano } \\
\hline & & & \multicolumn{2}{|c|}{ Hogares } & \multicolumn{2}{|c|}{ Individuos } & \multirow{2}{*}{\begin{tabular}{|c} 
Observaciones \\
(individuos)
\end{tabular}} & \multirow{2}{*}{$\begin{array}{l}\text { Población } \\
\text { expandida }\end{array}$} \\
\hline & & & Urbano & Nacional & Urbano & Nacional & & \\
\hline ENH (urbana) & 1992 & III Trimestre & 15,626 & & 69,683 & & $100 \%$ & $100 \%$ \\
\hline $\mathrm{ENH}$ & 1996 & III Trimestre & 22,897 & 31,264 & 99,409 & 137,423 & $72 \%$ & $61 \%$ \\
\hline MESEP & 2002 & Anual & 119,827 & 129,164 & 494,061 & 535,366 & $92 \%$ & $74 \%$ \\
\hline MESEP & 2005 & Anual & 134,628 & 151,402 & 530,678 & 602,988 & $88 \%$ & $75 \%$ \\
\hline MESEP & 2012 & Anual & 205,308 & 228,662 & 726,727 & 812,711 & $89 \%$ & $76 \%$ \\
\hline
\end{tabular}

\section{DESIGUALDAD DE INGRESO: HECHOS BÁSICOS Y FUENTES DE}

\section{CAMBIO}

Las dinámicas que rigen los cambios en la desigualdad se explican por un conjunto de fuerzas que pueden compensarse mutuamente. Esta sección se concentra en los principales

\footnotetext{
${ }^{10}$ Estos efectos se ven reflejados en el método de renta imputada por propiedad de la vivienda en la penúltima sección donde se realizan descomposiciones por fuentes de ingresos.
} 
determinantes de uno de los componentes del ingreso per cápita: el ingreso laboral individual. A continuación realizamos el análisis a profundidad por cada uno de dichos determinantes: los retornos a la educación, la brecha salarial entre hombres y mujeres, los retornos a la experiencia, los factores no observados, las horas de trabajo, el empleo y la estructura educativa.

\subsection{Retornos a la educación}

Un incremento en los retornos a la educación implica un aumento en la brecha de salarios entre trabajadores con mayores niveles de educación respecto de aquellos sin educación o con niveles menores. Una brecha más amplia, en principio, implica una mayor desigualdad de la distribución de ingreso de los hogares. La tabla 4.1 presenta el salario horario real por nivel educativo para los individuos de entre 12 y 65 años de edad. En línea con la evolución económica los salarios reales se incrementaron un 21\% entre 1992 y 1996, luego se reducen un $13 \%$ entre 1996 y 2002. Posteriormente se observa un descenso marginal (del orden del 2\%) hasta 2005 y una fuerte recuperación hacia 2012 (18\%). Los incrementos en los salarios promedio fueron para todos los niveles educativos entre 1992 y 1996, pero lo mayores incrementos los presentaron el grupo de educación superior incompleta y superior completa, mientras que para la segunda década (2002-2012) los incrementos más significativos fueron para los niveles educativos inferiores, hasta secundaria incompleta. 
Tabla 4.1. Salario horario por nivel educativo en Colombia, años seleccionados.

\begin{tabular}{|c|c|c|c|c|c|c|c|c|c|c|c|}
\hline \multirow{3}{*}{$\begin{array}{c}\text { Nivel } \\
\text { educativo }\end{array}$} & \multicolumn{6}{|c|}{ Salario Real (\$ 2008) } & \multirow{2}{*}{\multicolumn{5}{|c|}{ Cambio (porcentual) }} \\
\hline & \multicolumn{3}{|c|}{ Urbano } & \multicolumn{3}{|c|}{ Nacional } & & & & & \\
\hline & 1992 & 1996 & 2002 & 2002 & 2005 & 2012 & $92-96$ & $96-02$ & $02-05$ & 05-12 & $02-12$ \\
\hline $\begin{array}{l}\text { Primaria } \\
\text { incompleta }\end{array}$ & 2,353 & 2,754 & 2,097 & 1,787 & 1,788 & 2,174 & $17 \%$ & $-24 \%$ & $0 \%$ & $22 \%$ & $22 \%$ \\
\hline $\begin{array}{l}\text { Primaria } \\
\text { completa }\end{array}$ & 2,640 & 3,200 & 2,318 & 2,085 & 2,158 & 2,593 & $21 \%$ & $-28 \%$ & $4 \%$ & $20 \%$ & $24 \%$ \\
\hline $\begin{array}{l}\text { Secundaria } \\
\text { incompleta }\end{array}$ & 3,088 & 3,560 & 2,752 & 2,619 & 2,520 & 2,889 & $15 \%$ & $-23 \%$ & $-4 \%$ & $15 \%$ & $10 \%$ \\
\hline $\begin{array}{l}\text { Secundaria } \\
\text { completa }\end{array}$ & 4,012 & 4,831 & 3,468 & 3,397 & 3,232 & 3,612 & $20 \%$ & $-28 \%$ & $-5 \%$ & $12 \%$ & $6 \%$ \\
\hline $\begin{array}{l}\text { Superior } \\
\text { incompleta }\end{array}$ & 5,820 & 7,193 & 6,679 & 6,606 & 5,581 & 5,543 & $24 \%$ & $-7 \%$ & $-16 \%$ & $-1 \%$ & $-16 \%$ \\
\hline $\begin{array}{l}\text { Superior } \\
\text { completa }\end{array}$ & 10,151 & 13,740 & 12,462 & 12,344 & 11,441 & 13,511 & $35 \%$ & $-9 \%$ & $-7 \%$ & $18 \%$ & $9 \%$ \\
\hline Total & 4,129 & 4,986 & 4,348 & 3,763 & 3,685 & 4,338 & $21 \%$ & $-13 \%$ & $-2 \%$ & $18 \%$ & $15 \%$ \\
\hline
\end{tabular}

Nota: los datos cubren a trabajadores entre 12 y 65 años.

Fuente: Cálculos propios basados en la ENH-Urbana y MESEP.

La tabla 4.2 muestra los resultados de una ecuación del logaritmo del salario horario, estimado usando el proceso Heckman para corregir por sesgo de selección (la ecuación de selección se muestra en la tabla del anexo A8). Siguiendo a Gasparini et al. (2005) se incluye la edad y edad al cuadrado junto con variables dicotómicas que capturan el máximo nivel educativo alcanzado, el género y la condición de tener menos de 18 años. ${ }^{11}$ La ecuación de selección incluye además de las variables anteriores el número de hijos menores en el hogar, y variables dicotómicas para el estado civil, para asistencia educativa, y para la presencia de jefe o cónyuge ocupados en el hogar. Para los niveles educativos, la categoría omitida comprende a los individuos sin educación o con educación primaria incompleta.

\footnotetext{
${ }^{11}$ Para el caso de las encuestas MESEP que tienen una muestra a nivel nacional, éstas permiten agregar más variables dicotómicas para controlar si el individuo pertenece al sector urbano/rural y cinco variables que permiten identificar la región a la que pertenece el individuo. Para una clasificación de las regiones consistente con su estructura económica y poblacional se siguió la propuesta de Galvis, Luis A. (2007).
} 
Tabla 4.2. Ecuación del logaritmo del salario horario

\begin{tabular}{|c|c|c|c|c|c|c|}
\hline \multicolumn{3}{|c|}{ Encuesta Nacional de Hogares (urbano) } & \multicolumn{4}{|c|}{ MESEP } \\
\hline & 1992 & 1996 & & 2002 & 2005 & 2012 \\
\hline \multicolumn{3}{|l|}{ Log salario horario } & \multicolumn{4}{|l|}{ Log salario horario } \\
\hline Primaria completa & $\begin{array}{c}0.183^{\star * \star} \\
(10.32)\end{array}$ & $\begin{array}{c}0.183^{\star \star \star} \\
(7.4)\end{array}$ & Primaria completa & $\begin{array}{c}0.144^{\star \star \star} \\
(8.75)\end{array}$ & $\begin{array}{l}0.130^{\star \star \star} \\
(12.29)\end{array}$ & $\begin{array}{c}0.128^{\star \star \star} \\
(13.65)\end{array}$ \\
\hline Secundaria incompleta & $\begin{array}{l}0.393^{\star * \star} \\
(22.89)\end{array}$ & $\begin{array}{c}0.357^{\star * *} \\
(15.2)\end{array}$ & Secundaria incompleta & $\begin{array}{l}0.353^{\star * *} \\
(22.06)\end{array}$ & $\begin{array}{c}0.303^{\star * *} \\
(29.44)\end{array}$ & $\begin{array}{c}0.249^{\star * *} \\
(26.16)\end{array}$ \\
\hline Secundaria completa & $\begin{array}{c}0.644^{* * *} \\
(35.72)\end{array}$ & $\begin{array}{c}0.646^{\star * *} \\
(26.52)\end{array}$ & Secundaria completa & $\begin{array}{c}0.603^{\star \star *} \\
(38.47)\end{array}$ & $\begin{array}{c}0.510^{\star * *} \\
(49.69)\end{array}$ & $\begin{array}{c}0.446^{\star * *} \\
(51.15)\end{array}$ \\
\hline Superior incompleta & $\begin{array}{l}1.044^{\star \star *} \\
(45.88)\end{array}$ & $\begin{array}{l}1.089^{\star * *} \\
(37.52)\end{array}$ & Superior incompleta & $\begin{array}{l}1.182^{\star * *} \\
(63.38)\end{array}$ & $\begin{array}{c}0.995^{\star * *} \\
(80.63)\end{array}$ & $\begin{array}{c}0.867^{\star \star *} \\
(89.84)\end{array}$ \\
\hline Superior completa & $\begin{array}{c}1.513^{\star \star \star} \\
(70.24)\end{array}$ & $\begin{array}{c}1.638^{\star * *} \\
(56.77)\end{array}$ & Superior completa & $\begin{array}{c}1.712^{\star \star \star} \\
(96.29)\end{array}$ & $\begin{array}{l}1.614^{\star * *} \\
(137.57)\end{array}$ & $\begin{array}{l}1.628^{\star * *} \\
(160.4)\end{array}$ \\
\hline Hombre & $\begin{array}{l}0.191^{\star * \star} \\
(18.09)\end{array}$ & $\begin{array}{c}0.189^{\star \star \star} \\
(14.9)\end{array}$ & Hombre & $\begin{array}{c}0.112^{\star \star \star} \\
(12.16)\end{array}$ & $\begin{array}{c}0.163^{\star \star *} \\
(25.85)\end{array}$ & $\begin{array}{c}0.262^{* \star *} \\
(50.58)\end{array}$ \\
\hline Edad & $\begin{array}{c}0.0320^{* * *} \\
(9.65)\end{array}$ & $\begin{array}{c}0.0274^{* * *} \\
(6.19)\end{array}$ & Edad & $\begin{array}{c}0.0314^{* * *} \\
(11.43)\end{array}$ & $\begin{array}{c}0.0292^{* * *} \\
(16.18)\end{array}$ & $\begin{array}{c}0.0417^{* * *} \\
(28.46)\end{array}$ \\
\hline Edad al cuadrado & $\begin{array}{c}-0.000282^{* * *} \\
(6.39)\end{array}$ & $\begin{array}{c}-0.000187^{* * *} \\
(3.62)\end{array}$ & Edad al cuadrado & $\begin{array}{c}-0.000286^{* * *} \\
(8.18)\end{array}$ & $\begin{array}{c}-0.000272^{* * *} \\
(11.83)\end{array}$ & $\begin{array}{c}-0.000437^{\text {** }} \\
(23.85)\end{array}$ \\
\hline Menor de 18 & $\begin{array}{l}0.0331 \\
(0.89)\end{array}$ & $\begin{array}{l}0.139^{* *} \\
(3.11)\end{array}$ & Urbano & $\begin{array}{c}0.335^{* * *} \\
(21.95)\end{array}$ & $\begin{array}{c}0.343^{\star * *} \\
(36.9)\end{array}$ & $\begin{array}{c}0.334^{* * *} \\
(42.01)\end{array}$ \\
\hline Constante & $\begin{array}{c}4.829^{\star * *} \\
(74.51)\end{array}$ & $\begin{array}{c}5.762^{\star * \star} \\
(80.49)\end{array}$ & Región 02 & $\begin{array}{c}0.175^{\star * *} \\
(5.3)\end{array}$ & $\begin{array}{c}0.482^{\star \star \star} \\
(16.54)\end{array}$ & $\begin{array}{c}0.444^{* * *} \\
(19.15)\end{array}$ \\
\hline \multirow{3}{*}{$\begin{array}{l}\text { Observaciones } \\
\text { Chi } 2 \\
\text { Log Lik. } \\
\text { Rho } \\
\text { Sigma } \\
\text { Lambda }\end{array}$} & $\begin{array}{l}47915 \\
24.43\end{array}$ & $\begin{array}{l}67786 \\
55.08\end{array}$ & Región 03 & $\begin{array}{c}0.112^{\star \star \star} \\
(3.43)\end{array}$ & $\begin{array}{c}0.401^{\star * *} \\
(14.00)\end{array}$ & $\begin{array}{c}0.305^{\star * *} \\
(13.4)\end{array}$ \\
\hline & $\begin{array}{l}-7398332 \\
-0.125877\end{array}$ & $\begin{array}{l}-13749516 \\
-0.1723838\end{array}$ & Región 04 & $\begin{array}{c}-0.0302 \\
(0.92)\end{array}$ & $\begin{array}{c}0.267^{\star \star *} \\
(9.32)\end{array}$ & $\begin{array}{c}0.0881^{* * *} \\
(3.85)\end{array}$ \\
\hline & $\begin{array}{c}0.6271746 \\
-0.0789469\end{array}$ & $\begin{array}{l}0.6681869 \\
-0.1151846\end{array}$ & Región 05 & $\begin{array}{c}-0.0248 \\
(0.70)\end{array}$ & $\begin{array}{c}0.267^{* * *} \\
(9.03)\end{array}$ & $\begin{array}{c}0.132^{* * *} \\
(5.66)\end{array}$ \\
\hline \multicolumn{3}{|c|}{${ }^{*} p<0.05^{* *} p<0.01{ }^{* * *} p<0.001$} & Menor de 18 & $\begin{array}{c}-0.0308 \\
(0.95)\end{array}$ & $\begin{array}{c}-0.0189 \\
(0.84)\end{array}$ & $\begin{array}{c}-0.0621^{* *} \\
(3.01)\end{array}$ \\
\hline \multirow{8}{*}{\multicolumn{3}{|c|}{$\begin{array}{l}\text { Nota: los datos representan una estimación tipo } \\
\text { Heckman por máxima verosimilitud; los valores t están } \\
\text { entre paréntesis. Datos cubren a los individuos entre } 12 \\
\text { y } 65 \text { años de edad. } \\
\text { Fuente: Cálculos propios basados en la ENH-Urbana y } \\
\text { MESEP. }\end{array}$}} & Constante & $\begin{array}{c}5.723^{* * *} \\
(91.53) \\
\end{array}$ & $\begin{array}{l}5.688^{* * *} \\
(126.51) \\
\end{array}$ & $\begin{array}{c}6.006^{* * *} \\
(163.1)\end{array}$ \\
\hline & & & Observaciones & 352427 & 401805 & 548389 \\
\hline & & & Chi 2 & 166.69 & 230.43 & 397.92 \\
\hline & & & Log Lik. & $-2.86 \mathrm{E}+08$ & -24329589 & -29099986 \\
\hline & & & Rho & -0.1679381 & -0.1485477 & -0.1616643 \\
\hline & & & Sigma & 0.8084221 & 0.7415604 & 0.7570556 \\
\hline & & & Lambda & -0.1357648 & -0.1101571 & -0.1223889 \\
\hline & & & ${ }^{*} p<0.05^{* *}$ & $<0.001 "$ & & \\
\hline
\end{tabular}


Los coeficientes de la mayoría de los parámetros de los niveles educativos son positivos, estadísticamente significativos y crecientes con los niveles educativos, esto es, los retornos a la educación son siempre positivos. Para 1992, un individuo con educación primaria completa gana $18 \%$ más que un individuo con primaria incompleta. Similar situación se presenta si comparamos con secundaria incompleta, secundaria completa, superior incompleta y superior completa, con 39\%, 64\%, 104\% y $151 \%$ respectivamente. Es importante notar que a medida que se incrementa el nivel educativo, el diferencial salarial es mayor. Por ejemplo, con respecto al grupo tomado como base, una persona que ha logrado educación superior completa gana 46,9\% más que una con superior incompleta, mientras la diferencia entre secundaria completa e incompleta es del $25 \%$.

Al analizar el cambio de los coeficientes se observa que, con excepción del nivel primaria completa y secundaria incompleta, los retornos se incrementan durante el período 1992-1996 para todos los niveles educativos, siendo el cambio más importante para la categoría de educación superior completa (12.5\%). Para la siguiente década se observa una tendencia general hacia la reducción de los retornos a la educación, siendo las reducciones más importantes para los niveles de secundario completo y superior incompleto; y en una menor medida el nivel de superior completo, para el período 2002-2005. La tendencia continúa para el período 2005-2012 pero con reducciones un poco menores, y los retornos a la educación superior completa se incrementan marginalmente (1.4\%).

En síntesis, los retornos a la educación parecen que han tenido un efecto positivo en incrementar las brechas salariales para la década de los noventa, dado que los niveles educativos más altos fueron mejor remunerados que aquellos niveles educativos intermedios y bajos. Sin embargo, para la década del 2000, aunque los salarios reales se incrementan para todos los niveles educativos, ocurrieron mayores incrementos para los niveles educativos inferiores; generando, en principio, un efecto igualador. Es importante aclarar que el efecto final también dependerá de la composición de la estructura educativa.

\subsection{Brecha de género}

La tabla 4.3 presenta el salario horario promedio por género. El salario horario de los hombres relativo al de las mujeres es de 1.2 entre 1992 y 1996, luego los salarios relativos se equiparan para la década del 2000. 
Tabla 4.3. Salario horario por género en Colombia, años seleccionados

\begin{tabular}{lcccccc}
\hline \multirow{1}{*}{ Genero } & \multicolumn{6}{c}{ Salario Real (\$ 2008) } \\
\cline { 2 - 7 } & \multicolumn{5}{c}{ Urbano } & \multicolumn{3}{c}{ Nacional } \\
\cline { 2 - 7 } & $\mathbf{1 9 9 2}$ & $\mathbf{1 9 9 6}$ & $\mathbf{2 0 0 2}$ & $\mathbf{2 0 0 2}$ & $\mathbf{2 0 0 5}$ & $\mathbf{2 0 1 2}$ \\
\hline Mujeres & 3,690 & 4,406 & 4,281 & 3,972 & 3,766 & 4,286 \\
Hombres & 4,429 & 5,377 & 4,395 & 3,646 & 3,637 & 4,373 \\
Salario relativo & 1.20 & 1.22 & 1.03 & 0.92 & 0.97 & 1.02 \\
(hombres/mujeres) & 1.20 &
\end{tabular}

Nota: los datos cubren a trabajadores entre 12 y 65 años.

Fuente: Cálculos propios basados en la ENH-Urbana y MESEP.

El análisis condicionado muestra una leve reducción en los retornos por género para el período 1992-1996 (-0.2\%), pero un fuerte incremento para la década del 2000. Por ejemplo, un hombre en 2002 gana 11.2\% más que una mujer y en 2012 aumenta a 26.2\%; de hecho, el incremento de los retornos entre 2002-2012 es de 15 puntos porcentuales, lo que implica que se podría estar presentando un incremento de la brecha de género en ciertos niveles de la distribución del ingreso, por ejemplo, el caso de las mujeres más educadas, que no son captados con las estadísticas descriptivas de los salarios promedios.

Ahora bien, con respecto al efecto distributivo de la reducción de la brecha salarial por género, Gasparini et al. (2005) mencionan que éste depende de la posición relativa de las mujeres en la distribución del ingreso. Dado que generalmente las mujeres que trabajan se ubican en las partes superiores de la distribución, un incremento en los salarios de éstas relativo al de los hombres implicaría un incremento de la desigualdad. Por otro lado, un incremento de los salarios de las mujeres es más relevante en las partes inferiores de la distribución en donde una proporción importante de sus salarios van a los gastos del hogar, lo que se comprueba con la alta frecuencia de mujeres cabeza de hogar en los hogares pobres. Por lo que no se puede inferir a priori la dirección del efecto de la brecha salarial, sin simular el cambio en la desigualdad mediante la técnica de micro-descomposición.

\subsection{Retornos a la experiencia}

La edad es usada como proxy de la experiencia. La tabla 4.4 muestra el salario horario promedio para diferentes grupos de edad. En general, el perfil salario-edad muestra una forma cóncava, en la que los salarios se incrementan conforme aumenta la edad, hasta el grupo de los 30-39 años en 1992, en dónde comienza a declinar el salario horario promedio. La curva en 1996 presenta un punto de inflexión en el grupo de edad de 40-49 y para la década del 2000 se ubica en el grupo de 50-59. 
Tabla 4.4. Salario horario por grupos de edad, Colombia, años seleccionados

\begin{tabular}{crrrrrr}
\hline & \multicolumn{6}{c}{ Media (Col \$ 2008) } \\
\cline { 2 - 7 } Grupo etario & \multicolumn{4}{c}{ Urbano } & \multicolumn{4}{c}{ Nacional } \\
\cline { 2 - 7 } & $\mathbf{1 9 9 2}$ & $\mathbf{1 9 9 6}$ & $\mathbf{2 0 0 2}$ & $\mathbf{2 0 0 2}$ & $\mathbf{2 0 0 5}$ & $\mathbf{2 0 1 2}$ \\
\hline $12-19$ & 312 & 380 & 1580.3 & 1,288 & 1,333 & 1,506 \\
$20-29$ & 1,866 & 2,134 & 3284.9 & 2,847 & 2,836 & 3,531 \\
$30-39$ & 2,758 & 3,407 & 4293.1 & 3,787 & 3,723 & 4,560 \\
$40-49$ & 2,726 & 3,622 & 4873.7 & 4,289 & 4,113 & 4,588 \\
$50-59$ & 1,797 & 2,998 & 5252.8 & 4,432 & 4,281 & 4,811 \\
$60-65$ & 1,184 & 1,869 & 4800.7 & 3,794 & 3,755 & 4,091 \\
Total & 1,822 & 2,317 & 4115.8 & 3,519 & 3,475 & 4,105 \\
\hline
\end{tabular}

Nota: los datos cubren a trabajadores entre 12 y 65 años.

Fuente: Cálculos propios basados en la ENH-Urbana y MESEP.

Del análisis condicional observado en tabla 4.2, los coeficientes de la variable edad y edad al cuadrado sugieren que el perfil salario-edad también positivo y decreciente. Pero como se evidencia en el gráfico 4.1, hasta 2005 el punto de inflexión se ubica en niveles mayores de edad respecto de los observados en la tabla 4.4. Esta diferencia probablemente se explica porque los individuos con mayor edad tienen menores niveles educativos (al menos hasta 2005).

Hacia 2012 se incrementan los retornos (condicionales) y la concavidad es la más pronunciada de todos los períodos, con un punto de inflexión en los 48 años (menor al observado en el análisis no condicional). El efecto total sobre la desigualdad de la edad (o experiencia) en ambas décadas, dependerá de la composición y estructura etaria de los hogares a lo largo de la distribución del ingreso. 
Gráfico 4.1. Perfil de salario horario por grupos de edad para Colombia, años seleccionados

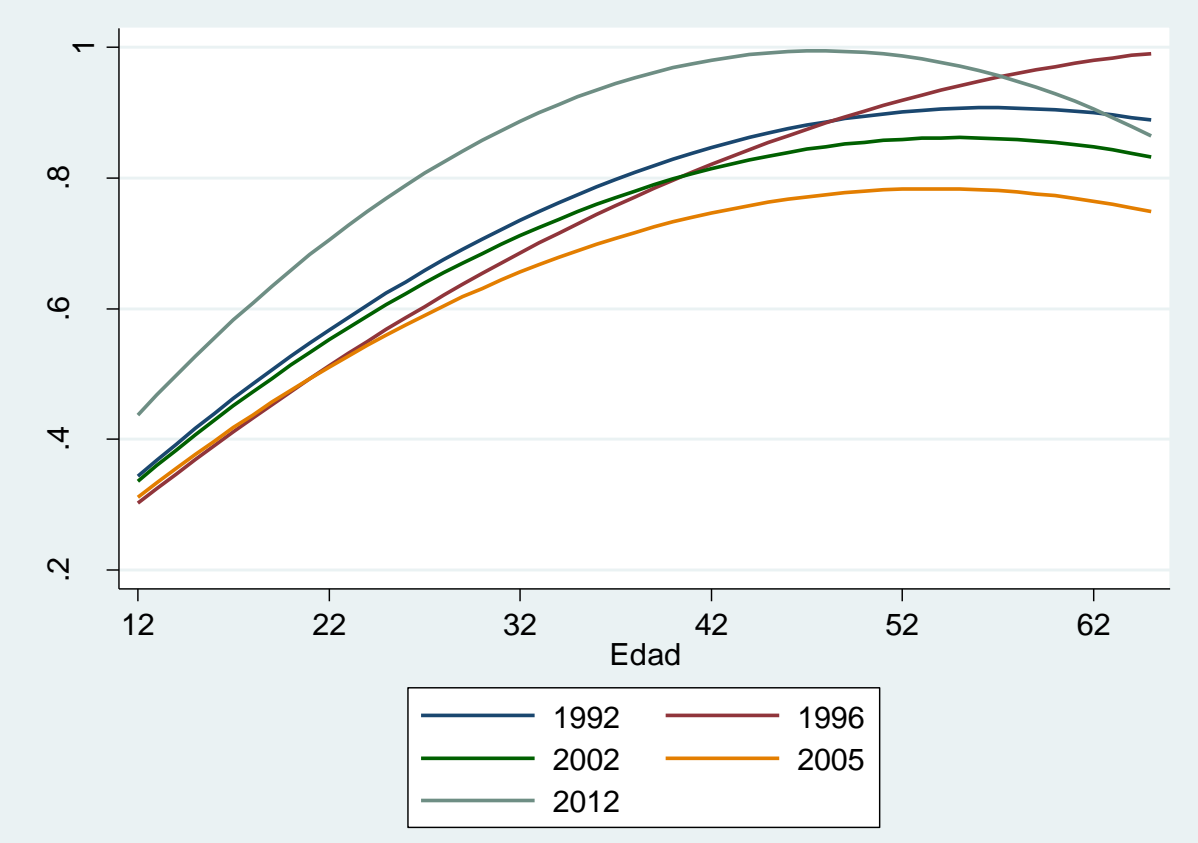

Fuente: salarios horarios estimados mediante el modelo de la tabla 4.2

\subsection{Factores no observados}

El término de error en la ecuación de salarios horario se interpreta como el efecto conjunto de las características y los retornos de los factores no observados, como por ejemplo la habilidad innata, calidad en la educación, cambios tecnológicos, etc. La varianza del error captura la contribución de la dispersión de los factores no observables en la desigualdad. La tabla 4.2 reporta la desviación estándar del término de error de cada ecuación del logaritmo del salario horario (etiquetada como sigma). Para la década de los noventa se observa un incremento en la desviación estándar, que pasa de 0.63 en 1992 a 0.67 en 1996, lo que implica, en principio, un efecto desigualador de los factores no observados. Durante la década del 2000 el desvío se reduce de 0.81 en 2002 a 0.74 en 2005 y luego se observa un ligero incremento en 2012 (0.75), lo que debería generar un efecto igualador para el período 2002-2012.

\subsection{Horas de trabajo}

Las horas de trabajo para el período bajo análisis presentan un incremento marginal para el período 1992-1996, luego disminuyen fuertemente (1.5 horas) entre 1996 y 2002. Para la década del 2000 hay un incremento (promedio) de una hora entre 2002 y 2005, y una reducción de media hora entre 2005 y 2012 (ver tabla 4.5). 
La tabla también muestra los promedios de las horas semanales de trabajo por nivel educativo; en ella se evidencian incrementos para los niveles educativos entre secundaria incompleta y superior incompleta, y reducciones para el resto de los niveles entre 1992 y 1996. Para el período 1996-2002 todos los grupos reducen sus horas, con excepción de secundaria completa; las reducciones más importantes se dan para los individuos que tienen educación (hasta) primaria incompleta y para el grupo de secundaria incompleta. Para la década del 2000 se presentan aumentos en las horas de trabajo para todos los niveles educativos entre 2002 y 2005, siendo importantes los cambios para los grupos de secundaria incompleta y superior incompleta. Posteriormente se presenta una disminución en las horas promedio para todos los grupos educativos, con excepción de superior incompleta entre 2005 y 2012.

Los patrones no son claros entre los grupos educativos como para inferir un efecto igualador o desigualador en las horas de trabajo para los períodos analizados, por lo que no es factible inferir una dirección particular a través del tiempo basado en el análisis no condicionado.

Tabla 4.5. Horas semanales por nivel educativo en Colombia, años seleccionados

\begin{tabular}{|c|c|c|c|c|c|c|c|c|c|c|c|}
\hline \multirow{3}{*}{$\begin{array}{c}\text { Nivel } \\
\text { educativo }\end{array}$} & \multicolumn{6}{|c|}{ Media } & \multirow{2}{*}{\multicolumn{5}{|c|}{ Cambio (porcentual) }} \\
\hline & \multicolumn{3}{|c|}{ Urbano } & \multicolumn{3}{|c|}{ Nacional } & & & & & \\
\hline & 1992 & 1996 & 2002 & 2002 & 2005 & 2012 & $92-96$ & $96-02$ & 02-05 & $05-12$ & $02-12$ \\
\hline $\begin{array}{l}\text { Primaria } \\
\text { incompleta }\end{array}$ & 48.8 & 48.5 & 45.6 & 44.4 & 44.8 & 44.6 & $-0.6 \%$ & $-6.1 \%$ & $0.9 \%$ & $-0.4 \%$ & $0.5 \%$ \\
\hline $\begin{array}{l}\text { Primaria } \\
\text { completa }\end{array}$ & 49.5 & 49.0 & 47.5 & 46.5 & 48.0 & 47.5 & $-1.0 \%$ & $-3.0 \%$ & $3.2 \%$ & $-1.0 \%$ & $2.2 \%$ \\
\hline $\begin{array}{l}\text { Secundaria } \\
\text { incompleta }\end{array}$ & 47.6 & 47.9 & 45.3 & 44.4 & 46.3 & 44.4 & $0.6 \%$ & $-5.4 \%$ & $4.3 \%$ & $-4.1 \%$ & $0.0 \%$ \\
\hline $\begin{array}{l}\text { Secundaria } \\
\text { completa }\end{array}$ & 47.4 & 48.0 & 48.0 & 47.6 & 49.2 & 48.9 & $1.3 \%$ & $0.0 \%$ & $3.4 \%$ & $-0.6 \%$ & $2.7 \%$ \\
\hline $\begin{array}{l}\text { Superior } \\
\text { incompleta }\end{array}$ & 43.1 & 43.7 & 42.5 & 42.4 & 44.8 & 45.3 & $1.4 \%$ & $-2.7 \%$ & $5.7 \%$ & $1.1 \%$ & $6.8 \%$ \\
\hline $\begin{array}{l}\text { Superior } \\
\text { completa }\end{array}$ & 45.5 & 45.4 & 44.4 & 44.3 & 45.4 & 44.4 & $-0.2 \%$ & $-2.2 \%$ & $2.5 \%$ & $-2.2 \%$ & $0.2 \%$ \\
\hline Total & 47.4 & 47.5 & 46.0 & 45.3 & 46.6 & 46.0 & $0.2 \%$ & $-3.2 \%$ & $2.9 \%$ & $-1.2 \%$ & $1.6 \%$ \\
\hline
\end{tabular}

Nota: los datos cubren a trabajadores entre 12 y 65 años.

Fuente: Cálculos propios basados en la ENH-Urbana y MESEP.

La tabla 4.6 muestra los resultados de las estimaciones de las horas de trabajo para los años seleccionados. Los coeficientes de las variables educativas son en su mayoría positivos y significativos, indicando que a mayores niveles educativos, la cantidad de horas 
trabajadas es mayor. Los coeficientes de edad son positivos y los de edad al cuadrado son negativos, sugiriendo un perfil de horas trabajadas de forma cóncava. Dado el coeficiente positivo de la variable hombre, los hombres (ceteris paribus) trabajan más horas que las mujeres para todos los años seleccionados. La tabla también evidencia un conjunto de variables que pueden afectar las decisiones de participación laboral del individuo: el estar casado, por ejemplo, tiende a disminuir sus horas de trabajo, pero si se incrementa el número de hijos aumentarán sus horas de trabajo; el ser menor de edad y/o estar asistiendo a algún nivel educativo tiende a disminuir (siempre ceteris paribus) las horas trabajadas.

Al igual que en el análisis de la tabla 4.5, al observar las diferencias entre los coeficientes de las variables educativas entre distintos momentos, tampoco surge un patrón uniforme que permita realizar hipótesis claras sobre la dirección de los efectos que produjeron los cambios en las horas trabajadas durante el período de análisis. 
Tabla 4.6. Ecuación de horas de trabajo

\begin{tabular}{|c|c|c|c|c|c|c|}
\hline \multicolumn{3}{|c|}{ TOBIT: ENH (Urbana) } & \multicolumn{4}{|c|}{ TOBIT: MESEP } \\
\hline & 1992 & 1996 & & 2002 & 2005 & 2012 \\
\hline Primaria completa & $\begin{array}{c}3.389^{* * *} \\
(6.44)\end{array}$ & $\begin{array}{l}1.190^{*} \\
(2.51)\end{array}$ & Primaria completa & $\begin{array}{c}1.574^{* * *} \\
(8.28)\end{array}$ & $\begin{array}{c}1.507^{* \star *} \\
(8.39)\end{array}$ & $\begin{array}{c}1.291^{* * *} \\
(8.24)\end{array}$ \\
\hline Secundaria incompleta & $\begin{array}{c}2.641^{* \star *} \\
(5.39)\end{array}$ & $\begin{array}{l}-0.415 \\
(0.94)\end{array}$ & Secundaria incompleta & $\begin{array}{l}0.160 \\
(0.85)\end{array}$ & $\begin{array}{c}0.908^{* * *} \\
(5.30)\end{array}$ & $\begin{array}{c}1.404^{* * *} \\
(9.43)\end{array}$ \\
\hline Secundaria completa & $\begin{array}{c}5.973^{\star \star *} \\
(11.26)\end{array}$ & $\begin{array}{c}4.474^{\star * *} \\
(9.68)\end{array}$ & Secundaria completa & $\begin{array}{c}3.496^{\star * *} \\
(17.95)\end{array}$ & $\begin{array}{c}3.546^{* * *} \\
(19.18)\end{array}$ & $\begin{array}{c}3.826^{* * *} \\
(25.64)\end{array}$ \\
\hline Superior incompleta & $\begin{array}{c}10.84^{\star * *} \\
(15.77)\end{array}$ & $\begin{array}{c}9.621^{* * *} \\
(15.40)\end{array}$ & Superior incompleta & $\begin{array}{c}5.321^{* * *} \\
(18.91)\end{array}$ & $\begin{array}{c}6.206^{* * *} \\
(25.46)\end{array}$ & $\begin{array}{c}6.346^{* * *} \\
(36.48)\end{array}$ \\
\hline Superior completa & $\begin{array}{c}6.769^{\star * *} \\
(10.76)\end{array}$ & $\begin{array}{c}6.388^{\star * *} \\
(11.11)\end{array}$ & Superior completa & $\begin{array}{c}5.170^{\star * *} \\
(21.07)\end{array}$ & $\begin{array}{c}4.902^{\star \star \star} \\
(21.89)\end{array}$ & $\begin{array}{c}4.906^{\star * *} \\
(26.40)\end{array}$ \\
\hline Hombre & $\begin{array}{c}24.12^{\star * *} \\
(69.80)\end{array}$ & $\begin{array}{c}24.44^{* * *} \\
(81.79)\end{array}$ & Hombre & $\begin{array}{l}23.01^{* * *} \\
(174.88)\end{array}$ & $\begin{array}{l}24.10^{* * *} \\
(202.05)\end{array}$ & $\begin{array}{l}21.36^{\star * *} \\
(233.46)\end{array}$ \\
\hline Edad & $\begin{array}{c}1.468^{\star \star \star} \\
(16.27)\end{array}$ & $\begin{array}{c}1.809^{\star \star *} \\
(23.31)\end{array}$ & Edad & $\begin{array}{c}1.575^{\star * *} \\
(47.15)\end{array}$ & $\begin{array}{l}1.803^{* * *} \\
(58.18)\end{array}$ & $\begin{array}{c}1.761^{* * *} \\
(72.78)\end{array}$ \\
\hline Edad al cuadrado & $\begin{array}{c}-0.0247^{* * *} \\
(21.77)\end{array}$ & $\begin{array}{c}-0.0294^{* * *} \\
(29.67)\end{array}$ & Edad al cuadrado & $\begin{array}{c}-0.0249^{* * *} \\
(58.72)\end{array}$ & $\begin{array}{c}-0.0280^{\star * *} \\
(71.34)\end{array}$ & $\begin{array}{c}-0.0256^{* * *} \\
(85.17)\end{array}$ \\
\hline Casado & $\begin{array}{c}-26.81^{* \star *} \\
(67.73)\end{array}$ & $\begin{array}{c}-25.61^{* * *} \\
(75.83)\end{array}$ & Urbano & $\begin{array}{c}-7.375^{\star \star \star} \\
(49.68)\end{array}$ & $\begin{array}{c}-5.048^{* * *} \\
(36.17)\end{array}$ & $\begin{array}{c}-3.388^{\star * \star} \\
(29.92)\end{array}$ \\
\hline Hijos menores & $\begin{array}{c}0.519^{\star * *} \\
(4.95)\end{array}$ & $\begin{array}{c}0.577^{\star * *} \\
(6.35)\end{array}$ & Región 02 & $\begin{array}{c}4.058^{\star * *} \\
(8.43)\end{array}$ & $\begin{array}{l}11.70^{* \star *} \\
(21.06)\end{array}$ & $\begin{array}{c}20.20^{\star * *} \\
(49.02)\end{array}$ \\
\hline Menor de 18 & $\begin{array}{c}-26.44^{\star * *} \\
(33.05)\end{array}$ & $\begin{array}{c}-26.02^{* * *} \\
(37.25)\end{array}$ & Región 03 & $\begin{array}{c}-2.419^{* * *} \\
(5.18)\end{array}$ & $\begin{array}{c}6.394^{* * *} \\
(11.76)\end{array}$ & $\begin{array}{c}14.84^{* * *} \\
(36.82)\end{array}$ \\
\hline Asiste & $\begin{array}{c}-30.78^{\star * *} \\
(51.30)\end{array}$ & $\begin{array}{c}-28.26^{* * *} \\
(53.54)\end{array}$ & Región 04 & $\begin{array}{c}-5.219^{\star * *} \\
(11.02)\end{array}$ & $\begin{array}{c}2.046^{* * *} \\
(3.73)\end{array}$ & $\begin{array}{c}11.65^{\star \star \star} \\
(28.66)\end{array}$ \\
\hline Jefe ocupado & $\begin{array}{l}44.99^{\star \star *} \\
(106.21)\end{array}$ & $\begin{array}{l}44.54^{* * *} \\
(125.52)\end{array}$ & Región 05 & $\begin{array}{c}-1.990^{* * *} \\
(4.10)\end{array}$ & $\begin{array}{c}5.147^{* * *} \\
(9.27)\end{array}$ & $\begin{array}{c}8.559^{* * *} \\
(20.66)\end{array}$ \\
\hline Cónyuge ocupado & $\begin{array}{l}59.96^{* * *} \\
(104.30)\end{array}$ & $\begin{array}{l}59.74^{* * *} \\
(118.79)\end{array}$ & Casado & $\begin{array}{l}-20.19^{\star * *} \\
(134.09)\end{array}$ & $\begin{array}{l}-20.37^{* * *} \\
(149.88)\end{array}$ & $\begin{array}{l}-16.49^{\star \star *} \\
(157.39)\end{array}$ \\
\hline Constante & $\begin{array}{c}-14.10^{* \star *} \\
(8.46)\end{array}$ & $\begin{array}{c}-19.14^{* * *} \\
(13.39)\end{array}$ & Hijos menores & $\begin{array}{c}1.203^{* * *} \\
(31.16)\end{array}$ & $\begin{array}{l}1.239^{* * *} \\
(34.11)\end{array}$ & $\begin{array}{c}0.922^{* * *} \\
(28.06)\end{array}$ \\
\hline $\begin{array}{l}\text { Observaciones } \\
\text { Censurados }\end{array}$ & $\begin{array}{l}47744 \\
22187\end{array}$ & $\begin{array}{l}67684 \\
32785\end{array}$ & Menor de 18 & $\begin{array}{c}-17.56^{\star \star *} \\
(59.33)\end{array}$ & $\begin{array}{c}-19.04^{\star * *} \\
(68.23)\end{array}$ & $\begin{array}{c}-22.15^{\star * *} \\
(98.96)\end{array}$ \\
\hline $\begin{array}{l}\text { Chi2 } \\
\text { Log Lik }\end{array}$ & $\begin{array}{r}39765.47 \\
-132453.25\end{array}$ & $\begin{array}{r}52512.45 \\
-188994.79\end{array}$ & Asiste & $\begin{array}{l}-24.55^{\star \star *} \\
(100.11)\end{array}$ & $\begin{array}{l}-26.69^{* * *} \\
(120.11)\end{array}$ & $\begin{array}{l}-16.78^{* * *} \\
(108.29)\end{array}$ \\
\hline $\begin{array}{l}\text { Pseudo R2 } \\
\text { Sigma }\end{array}$ & $\begin{array}{r}0.1305 \\
26.74768\end{array}$ & $\begin{array}{r}0.122 \\
27.33381\end{array}$ & Jefe ocupado & $\begin{array}{l}45.03^{\star * *} \\
(284.22)\end{array}$ & $\begin{array}{l}43.67^{* * *} \\
(302.02)\end{array}$ & $\begin{array}{l}39.37^{\star \star *} \\
(357.52)\end{array}$ \\
\hline \multirow{2}{*}{\multicolumn{3}{|c|}{$\begin{array}{l}\text { Nota: los datos representan la estimación de un } \\
\text { modelo Tobit por máxima verosimilitud, los valores t } \\
\text { están entre paréntesis. Los datos cubren a los } \\
\text { individuos entre } 12 \text { y } 65 \text { años. }\end{array}$}} & $\begin{array}{l}\text { Cónyuge ocupado } \\
\text { Constante }\end{array}$ & $\begin{array}{c}54.12^{* * *} \\
(249.54) \\
-13.88^{* * *} \\
(18.19)\end{array}$ & $\begin{array}{c}54.80^{* * *} \\
(278.70) \\
-26.12^{* * *} \\
(33.69)\end{array}$ & $\begin{array}{c}46.05^{\star * *} \\
(312.31) \\
-33.77^{\star * *} \\
(56.45)\end{array}$ \\
\hline & & & Observaciones & 35242 & 401795 & 548364 \\
\hline
\end{tabular}




\begin{tabular}{|lrrr|} 
Censurados & 159593 & 180442 & 223815 \\
Chi2 & 236911.5 & 293352.21 & 389477.87 \\
Log Lik & -1079856.1 & -1209104 & -1766608.5 \\
Pseudo R2 & 0.0989 & 0.1082 & 0.0993 \\
Sigma & 28.39563 & 28.11103 & 26.89314 \\
\hline
\end{tabular}

Adicionalmente, los gráficos 4.2 y 4.3 presentan las estimaciones de las horas de trabajo por grupos educativos para jefes de hogar y cónyuges, estimados con los parámetros del modelo Tobit de la tabla 4.6, en las cuales se aprecia una mayor variabilidad para las horas de trabajo de las mujeres cónyuges en relación con las horas de los hombres, que presentan una mayor estabilidad durante el período bajo análisis.

Gráfico 4.2. Horas semanales por nivel educativo hombres (Jefes de hogar), edad 40 años

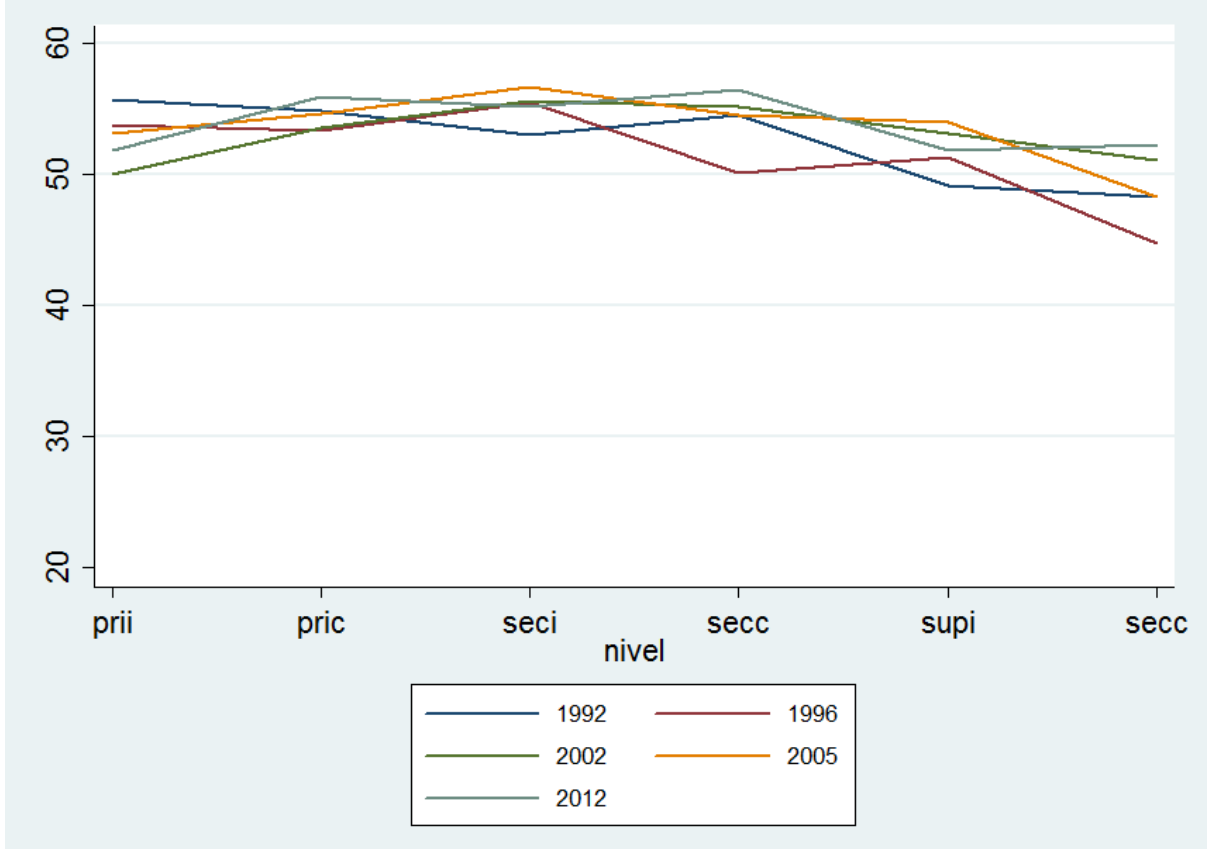

Fuente: salarios horarios estimados mediante el modelo de la tabla 4.6 


\section{Gráfico 4.3. Horas semanales por nivel educativo mujeres (Cónyuges), 40 años}

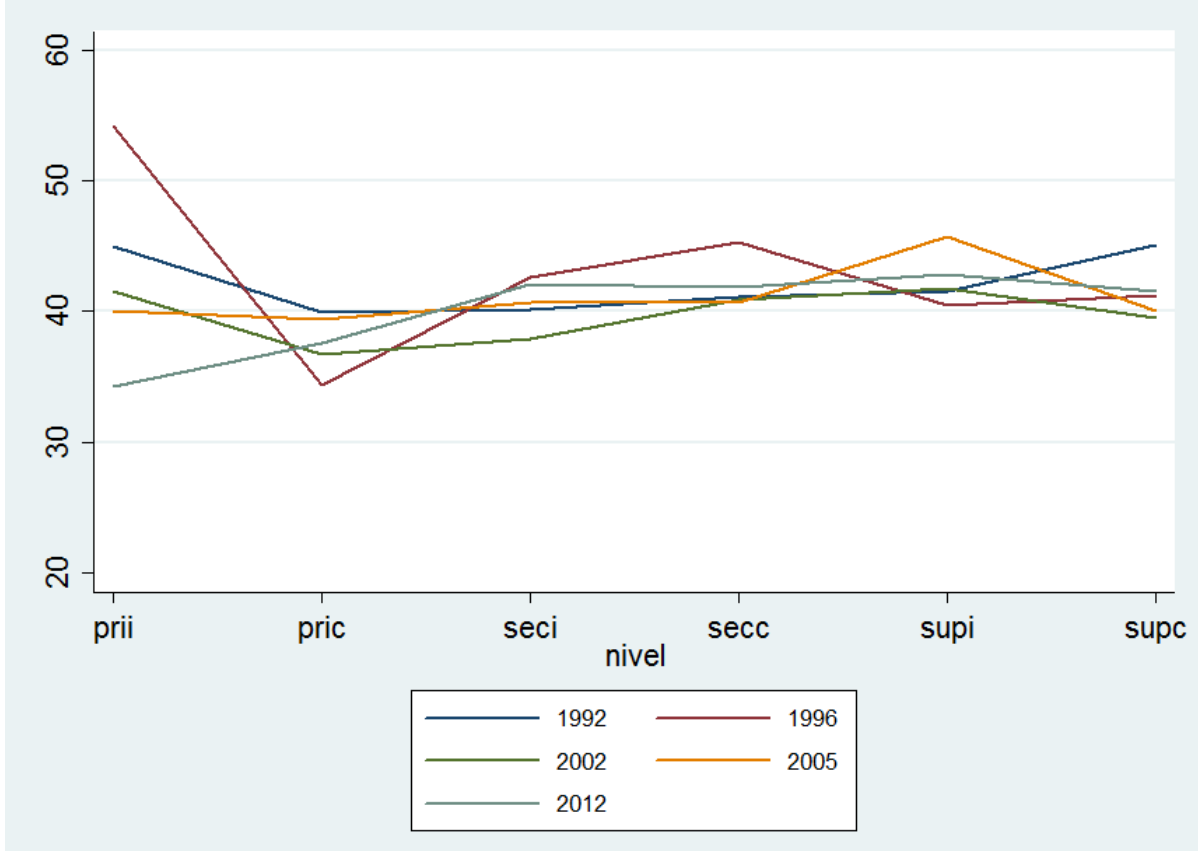

Fuente: salarios horarios estimados mediante el modelo de la tabla 4.6

\subsection{Empleo}

En la tabla 4.7, los individuos son clasificados de acuerdo con su rol en el hogar y de acuerdo con sus estatus ocupacional (empleados, desempleados o inactivos). La tasa de desempleo presenta un fuerte incremento del 5.8\% en 1992 al 11.7\% en 2002 (para el área urbana), y luego disminuye durante la siguiente década del $10.6 \%$ en 2002 al $6.3 \%$ en 2012. La composición de la estructura del mercado laboral para el período 1992-2002 muestra reducciones fuertes de la ocupación de los jefes de hogar que pasaron directamente al desempleo (o salidas desde la inactividad a otras categorías laborales), que tienden a ser compensados por la participación de los cónyuges que pasaron de la inactividad a emplearse o al desempleo. Los otros miembros del hogar reducen levemente su participación en el empleo, pero fueron importantes las proporciones que pasaron de la inactividad al desempleo. 
Tabla 4.7. Estructura laboral por rol en el hogar en Colombia.

\begin{tabular}{lcccccc}
\hline \multirow{2}{*}{$\begin{array}{l}\text { Estatus } \\
\text { Laboral }\end{array}$} & \multicolumn{5}{c}{ Urbano } & \multicolumn{3}{c}{ Nacional } \\
\cline { 2 - 6 } & $\mathbf{1 9 9 2}$ & 1996 & $\mathbf{2 0 0 2}$ & $\mathbf{2 0 0 2}$ & $\mathbf{2 0 0 5}$ & $\mathbf{2 0 1 2}$ \\
\hline Todos & & & & & & \\
Empleado & $54.9 \%$ & $52.6 \%$ & $54.7 \%$ & $55.2 \%$ & $56.3 \%$ & $61.4 \%$ \\
Desempleado & $5.8 \%$ & $7.4 \%$ & $11.7 \%$ & $10.6 \%$ & $7.9 \%$ & $7.3 \%$ \\
Inactivo & $39.3 \%$ & $45.3 \%$ & $33.6 \%$ & $34.1 \%$ & $35.8 \%$ & $31.3 \%$ \\
Jefe de Hogar & & & & & & \\
Empleado & $85.9 \%$ & $83.2 \%$ & $80.8 \%$ & $82.5 \%$ & $84.4 \%$ & $85.1 \%$ \\
Desempleado & $2.6 \%$ & $4.5 \%$ & $8.1 \%$ & $7.3 \%$ & $4.8 \%$ & $5.0 \%$ \\
Inactivo & $11.5 \%$ & $15.4 \%$ & $11.1 \%$ & $10.3 \%$ & $10.8 \%$ & $9.9 \%$ \\
Cónyuge & & & & & & \\
Empleado & $40.9 \%$ & $39.9 \%$ & $48.6 \%$ & $45.0 \%$ & $46.7 \%$ & $55.6 \%$ \\
Desempleado & $5.4 \%$ & $6.1 \%$ & $10.6 \%$ & $9.4 \%$ & $6.7 \%$ & $7.0 \%$ \\
Inactivo & $53.6 \%$ & $61.8 \%$ & $40.8 \%$ & $45.7 \%$ & $46.6 \%$ & $37.4 \%$ \\
Otros & & & & & & \\
Empleado & $42.5 \%$ & $39.8 \%$ & $40.1 \%$ & $42.1 \%$ & $42.1 \%$ & $45.3 \%$ \\
Desempleado & $7.9 \%$ & $9.7 \%$ & $14.6 \%$ & $13.5 \%$ & $10.5 \%$ & $9.4 \%$ \\
Inactivo & $49.6 \%$ & $55.9 \%$ & $45.2 \%$ & $44.4 \%$ & $47.3 \%$ & $45.3 \%$ \\
\hline
\end{tabular}

Nota: los datos cubren a trabajadores entre 12 y 65 años.

Fuente: Cálculos propios basados en la ENH-Urbana y MESEP.

Para la década 2002-2012 se presentan incrementos importantes en la proporción de empleados para todas las categorías de rol en el hogar y disminuciones en la proporción de desocupados e inactivos, lo que evidencia una recuperación del mercado laboral durante la década. También es importante señalar el fuerte incremento de la ocupación de los cónyuges (10.6\% para este período).

\subsection{Educación}

La tabla 4.8 presenta la estructura educativa en Colombia para los distintos períodos analizados, en donde se observa la participación de la población por nivel educativo. Se evidencia que entre 1992 y 1996 se presenta una reducción para los grupos con educación primaria y con educación superior, junto con el incremento en los niveles intermedios (secundaria). La tendencia a lo largo de la década del 2000 es una disminución en la participación de los niveles educativos de primarios (hasta secundaria incompleta) entre 2005 y 2012, y un fuerte incremento en la participación del grupo superior incompleta, seguido de secundaria completa. Estas tendencias permiten inferir un efecto igualador para la década de los noventa, y un efecto desigualador importante de los cambios en la estructura educativa para el período 2002-2012. 
Tabla 4.8. Composición de la muestra por nivel educativo en Colombia, años seleccionados

\begin{tabular}{lcccccc}
\hline & \multicolumn{3}{c|}{ Urbano } & \multicolumn{3}{c}{ Nacional } \\
\cline { 2 - 7 } $\begin{array}{l}\text { Nivel } \\
\text { Educativo }\end{array}$ & 1992 & 1996 & $\mathbf{2 0 0 2}$ & $\mathbf{2 0 0 2}$ & $\mathbf{2 0 0 5}$ & $\mathbf{2 0 1 2}$ \\
\hline $\begin{array}{l}\text { Primaria } \\
\text { incompleta }\end{array}$ & $13.8 \%$ & $12.5 \%$ & $11.6 \%$ & $17.4 \%$ & $17.7 \%$ & $13.6 \%$ \\
$\begin{array}{l}\text { Primaria } \\
\text { completa }\end{array}$ & $15.7 \%$ & $15.3 \%$ & $14.0 \%$ & $16.7 \%$ & $15.5 \%$ & $12.6 \%$ \\
$\begin{array}{l}\text { Secundaria } \\
\text { incompleta }\end{array}$ & $37.4 \%$ & $38.0 \%$ & $32.5 \%$ & $30.6 \%$ & $31.1 \%$ & $29.8 \%$ \\
$\begin{array}{l}\text { Secundaria } \\
\text { completa }\end{array}$ & $16.6 \%$ & $18.9 \%$ & $23.6 \%$ & $20.4 \%$ & $18.9 \%$ & $21.9 \%$ \\
$\begin{array}{l}\text { Superior } \\
\text { incompleta }\end{array}$ & $9.2 \%$ & $8.9 \%$ & $9.5 \%$ & $7.7 \%$ & $9.1 \%$ & $14.5 \%$ \\
$\begin{array}{l}\text { Superior } \\
\text { completa }\end{array}$ & $7.3 \%$ & $6.4 \%$ & $8.8 \%$ & $7.1 \%$ & $7.8 \%$ & $7.6 \%$ \\
\hline
\end{tabular}

Nota: los datos cubren a trabajadores entre 12 y 65 años.

Fuente: Cálculos propios basados en la ENH-Urbana y MESEP.

Con respecto a la estructura educativa, Gasparini et al. (2005) analizan que el efecto en la desigualdad general se puede evaluar mediante los efectos en la desigualdad entre grupos y la desigualdad ponderada dentro de los grupos. El caso de Colombia, al igual que el de varios países en Latinoamérica en la década del 2000, la estructura educativa cambia a favor del grupo con una distribución de ingresos que se caracteriza por tener: (a) salarios medios mayores con respecto a los otros grupos; y (b) una alta dispersión salarial. Algunos autores atribuyen dicha dispersión salarial en el grupo con educación superior a factores de calidad en las universidades del país (ver Posso, 2008). Los cambios en la estructura educativa permiten afirmar inicialmente un efecto desigualador en términos distributivos durante la última década, pero el efecto final depende también de la importante reducción de los retornos a la educación durante el mismo período (ver sección 4.1) y de la composición y estructura de los hogares.

\section{DESCOMPOSICIÓN MICROECONOMÉTRICA DEL COEFICIENTE DE} GINI

La metodología de descomposiciones microeconométricas es utilizada para la descomposición de la desigualdad entre los diferentes factores que la afectan. La estrategia 
que se aplica en esta sección es una versión simplificada de la aplicada por Gasparini et al. (2005). ${ }^{12}$ La simplificación radica en que el análisis de los cambios en la desigualdad se limita al coeficiente de Gini del ingreso per cápita familiar, y las estimaciones de salarios y horas trabajadas se estiman en forma conjunta para todos los individuos en cada uno de los años bajo análisis.

\subsection{Metodología}

A continuación se sigue a grandes rasgos la presentación de Gasparini et al. (2005).

Teniendo en cuenta que el ingreso laboral del individuo $i$ en el momento $t, Y_{i t}$, es función de los vectores que contienen las características observables que afectan los salarios y el empleo, $X_{i t}$, del vector $\beta_{i t}$ de parámetros que determinan el salario horario de mercado, de los parámetros que afectan los resultados en el empleo (la participación y las horas de trabajo), $\lambda_{i t}$, y de las características no observables, $\varepsilon_{i t}$. El ingreso laboral se puede expresar de la siguiente manera:

$$
\text { 1. } Y_{i t}=F\left(X_{i t}, \beta_{i t}, \lambda_{i t}, \varepsilon_{i t}\right) ; \quad i=1,2, \ldots, N
$$

Con la ecuación anterior se puede simular el ingreso laboral individual, es decir, el investigador puede cambiar cualquier parámetro de la ecuación 1 con el que se desee trabajar de acuerdo con el objetivo que persiga. Por ejemplo, se puede obtener la siguiente expresión:

\section{2. $Y_{i t}\left(\beta_{i t \prime}\right)=F\left(X_{i t}, \beta_{i t,}, \lambda_{i t}, \varepsilon_{i t}\right)$}

que representa el ingreso laboral que el individuo $i$ debería haber obtenido en el momento $t$ si los parámetros que determinan los salarios hubieran sido los del tiempo $t^{\prime}$, manteniendo lo demás constante. De manera general, se puede definir el ingreso laboral simulado $Y_{i t}\left(k_{t}\right)$, donde $k$ representa un parámetro o conjunto de parámetros representados en la ecuación 1.

Como es de esperarse, el ingreso laboral es la principal fuente de ingresos de los hogares colombianos (como veremos en la penúltima sección) pero no es la única. Adicionalmente, para estudiar los cambios en el bienestar y más específicamente en la desigualdad, es conveniente utilizar una medida de ingreso que aproxime mejor el bienestar

\footnotetext{
${ }^{12}$ Basada en Bourguignon, et. al. (1998).
} 
de los individuos. Por simplicidad podemos tomar como medida el ingreso per cápita familiar. Para el individuo $i$ perteneciente a la familia $h$ el ingreso per cápita familiar en el momento $t$ será:

$$
\text { 3. } Y_{i t}^{p c} \equiv Y_{i h t}^{p c}=\frac{\sum_{j=1}^{n_{h}}\left(Y_{j t}+Y_{j t}^{0}\right)}{n_{h}}
$$

Donde $n_{h}$ es el número de miembros del hogar $h$ (al que pertenece el individuo) y $\sum_{j=1}^{n} Y_{j t}^{0}$ es el total otros ingresos (distintos a los laborales) del hogar $h$ (que se mantienen constantes en la simulación). En consecuencia el ingreso per cápita familiar simulado será:

4. $Y_{i t}^{p c}\left(k_{t \prime}\right) \equiv Y_{i h t}^{p c}\left(k_{t \prime}\right)=\frac{\sum_{j=1}^{n_{h}}\left(Y_{j t}\left(k_{t \prime}\right)+Y_{j t}^{0}\right)}{n_{h}}$

Si $N$ es la población total, la distribución del ingreso per cápita familiar puede representarse de la siguiente manera:

5. $D_{t}^{p c}=\left\{Y_{1 t}^{p c}, Y_{2 t}^{p c}, \ldots, Y_{N t}^{p c}\right\}$

Por lo tanto, la distribución simulada será:

$$
\text { 6. } D_{t}^{p c}\left(k_{t \prime}\right)=\left\{Y_{1 t}^{p c}\left(k_{t}\right), Y_{2 t}^{p c}\left(k_{t \prime}\right), \ldots, Y_{N t}^{p c}\left(k_{t \prime}\right)\right\}
$$

La contribución que realiza al cambio total en la distribución de un cambio en $k$ entre $t$ y $t^{\prime}$, manteniendo todo lo demás constante, puede obtenerse comparando las ecuaciones 5 y 6. Siguiendo a Gasparini et al (2005), se compara un índice de Gini $G(D)$ de la función de distribución.

7. $G\left[D_{t}^{p c}\left(k_{t}\right)\right]-G\left(D_{t}^{p c}\right)$

A partir de la ecuación 7 es posible computar los siguientes efectos:

- Retornos a la educación $\left(k=\beta^{e d}\right)$ : mide el efecto de cambios en los parámetros que relacionan la educación con el salario horario en la desigualdad.

- Brecha salarial de género $\left(k=\beta^{g}\right)$ : mide el efecto de los cambios en los parámetros que relacionan el género con el salario horario en la desigualdad.

- Retornos a la experiencia $\left(k=\beta^{\text {ex }}\right)$ : mide el efecto de los cambios en los parámetros que relacionan la experiencia (o edad) con el salario horario en la desigualdad. 
- Dotaciones y retornos de factores no observados $\left(k=\varepsilon^{w}\right)$ : mide el efecto de cambios en los factores inobservables y sus remuneraciones que afectan el salario horario en la desigualdad.

- Horas de trabajo y empleo $(k=\lambda)$ : mide el efecto de cambios en los parámetros que determinan las horas de trabajo y la participación en el mercado laboral en la desigualdad.

- Educación $\left(k=X^{e d}\right)$ : mide el efecto de los cambios en la estructura educativa de la población en la desigualdad.

La metodología propuesta tiene la limitación de que la estructura sociodemográfica y de ingresos permanece inalterada; además, no se pueden analizar los cambios de segundo orden, como sería el caso de un modelo de equilibrio general. Cabe resaltar que en este documento no se van a tener en cuenta otras características que son inherentes a la distribución del ingreso como la fecundidad y la composición del hogar, entre otros, que bien podrían extenderse con esta metodología. También para este ejercicio se consideran constantes los ingresos individuales de fuentes no laborales, la renta implícita de la vivienda propia y para las encuestas MESEP los ajustes de armonización, que no pueden desagregarse por fuente de ingreso.

\subsection{Estrategia de estimación}

Para poder simular la distribución del ingreso individual se debe plantear una forma funcional para la ecuación 1 con el objeto de obtener estimaciones de los parámetros $\beta, \lambda$ y el término de error $\varepsilon$. A continuación se explica la estrategia para solucionar este problema, propuesta por Gasparini et al. (2005).

Sea $L_{i}$ denota el número de horas de trabajadas por la persona $i$ y $w_{i}$ es el salario horario recibido. El ingreso laboral está dado por $Y_{i}=L_{i} w_{i}$. El número de horas de trabajo $L_{i}$ provienen de un proceso de maximización de la utilidad que determina la participación óptima en el mercado de trabajo, mientras que los salarios son determinados por fuerzas del mercado.

La especificación econométrica es la usada por Gasparini et al. (2005), la cual corresponde a una forma reducida de las decisiones laborales propuesto por Heckman (1974). En ésta se plantea la especificación de dos ecuaciones, la primera para los salarios 
horarios $w_{i}$, ${ }^{13}$ en función de un conjunto de características $X_{1 i}$, y la segunda para el número de horas trabajadas $L_{i}$ en función de las características $X_{2 i}$. Los términos error $\varepsilon_{1 i}$ y $\varepsilon_{2 i}$ de cada ecuación representan los factores inobservables que afectan la determinación de las variables endógenas. Lo anterior puede expresarse de la siguiente forma:

8. $w_{i}^{*}=X_{1 i} \beta+\varepsilon_{1 i}, \quad i=1,2, \ldots, N$

9. $L_{i}^{*}=X_{2 i} \lambda+\varepsilon_{2 i}$,

Con,

$$
\begin{array}{ll}
w_{i}=w_{i}^{*} & \text { si } L_{i}^{*}>0 \\
w_{i}=0 & \text { si } L_{i}^{*} \leq 0 \\
L_{i}=L_{i}^{*} & \text { si } L_{i}^{*}>0 \\
L_{i}=0 & \text { si } L_{i}^{*} \leq 0
\end{array}
$$

Donde $w_{i}$ y $L_{i}$ es el valor observado de los salarios y las horas trabajadas respectivamente. Esta notación enfatiza en que, consistentemente con los datos usados para la estimación, los salarios observados para un individuo que no trabaja son cero.

Adicionalmente se asume que los términos error $\varepsilon_{1 i}$ y $\varepsilon_{2 i}$ tienen una distribución normal bivariada, donde $E\left(\varepsilon_{1 i}\right)=E\left(\varepsilon_{2 i}\right)=0$, varianzas $\sigma_{1}^{2}$ y $\sigma_{2}^{2}$ y coeficiente de correlación $\rho$. Para la estimación de $w_{i}$ se utiliza el método de Heckman estimado por máxima verosimilitud, y en el caso de $L_{i}$ se estima un modelo Tobit estándar para datos censurados.

\section{Estructura educativa}

Adicional a los retornos a la educación y a la cantidad de horas trabajadas, se simulan los cambios en la estructura educativa de la población y su efecto sobre la distribución del ingreso. Para ello Gasparini et al. (2005) establecen un mecanismo no paramétrico que consiste en dividir la población adulta en diez grupos homogéneos por género y edad, para luego replicar la estructura educativa de cada celda en el año $t^{\prime}$ en la celda correspondiente al año $t$. El objetivo es intentar calcular qué es lo que sucede con la distribución del ingreso en el período $t$ si tuviera la estructura educativa de otro período $t^{\prime}$.

\footnotetext{
${ }^{13}$ En la estimación concreta se estima el logaritmo de los salarios horarios.
} 


\section{Dispersión de los inobservables}

Las características no observables que afectan los salarios son modeladas como el término de error de la ecuación de regresión de salarios (ecuación 8). Su media es trivialmente normalizada en cero y sus varianzas son estimadas como un parámetro extra en el proceso Heckman. En orden de simular los efectos de los cambios en los inobservables entre $t$ y $t^{\prime}$ en la desigualdad, los residuos estimados de la ecuación de salarios del año $t$ son reescalados por $\sigma_{t \prime} / \sigma_{t}$, donde $\sigma$ es la desviación estándar estimada de la ecuación de salarios. En consecuencia, es posible interpretar el efecto de los cambios en los factores inobservables como un cambio en la dispersión de los mismos.

\section{Horas trabajadas y estructura de empleo}

Siguiendo a Gasparini et al. (2005) se divide el efecto sobre la desigualdad de cambios en el empleo mediante tres simulaciones; por un lado, se simula el efecto agregado de los cambios en el empleo; por el otro, se simulan separadamente los efectos de las horas trabajadas y de la participación en el mercado laboral.

Para estimar el efecto empleo y el efecto participación, se debe considerar el cambio para todos los individuos. Para los individuos que trabajan tanto en el año $t$ como en el año $t^{\prime}$, en el cómputo del efecto empleo se asignan los salarios observados en el año $t$ y las

horas simuladas en el año $t^{\prime}$. Para el cómputo del efecto participación, en cambio, se asignan las horas observadas en el año $t$. En el caso de las personas con horas simuladas negativas en ambos casos se asignan ingresos (y participación) iguales a cero.

Adicionalmente el cómputo de los efectos empleo y participación surge el problema de tener que incluir ingresos de individuos que no trabajan en el año $t$ pero que resultaron con estimaciones positivas de horas de trabajo según los parámetros del año $t^{\prime}$. En este caso es necesario simular los salarios que tendrían en el período $t$. Para ello se simulan los ingresos y las horas basadas en las estimaciones de las ecuaciones 8 y 9 correspondientes al año $t$, siempre incluyendo los residuos estimados a partir de la distribución normal bivariada. Si en la simulación se obtienen horas trabajadas positivas para el año $t$ (año en el que no trabajan) esto es inconsistente con lo observado ese período, por lo que se realiza un 
proceso de remuestreo de los términos de error hasta obtener predicciones consistentes (horas trabajadas negativas). ${ }^{14}$

Para el efecto horas de trabajo simplemente se simula la cantidad de horas trabajadas utilizando los parámetros de la ecuación 9 y se ignora a aquellos individuos que cambian de estado en la simulación. En este cálculo sólo se considera el cambio en las horas trabajadas de los individuos que efectivamente trabajan y que también lo hacen en la simulación.

\subsection{Resultados}

En esta sub-sección se muestran los resultados obtenidos con la metodología propuesta. El objetivo es mostrar la relevancia cuantitativa de los determinantes anteriormente discutidos sobre los cambios en la desigualdad en Colombia durante 19922012. Es necesario notar que el método presenta "path dependent". Esto significa que dependiendo del período base que se tome, entre $t$ y $t^{\prime}$, los resultados no serán los mismos. Por lo tanto, es necesario realizar el ejercicio dos veces, para cada período tomado como base y posteriormente realizar un promedio de los cambios ocurridos entre ambos períodos.

Las tablas del anexo A.2 a A.5 presentan los resultados de tomar como año base a los $t$ y $t^{\prime}$. La tabla 5.1 reporta los promedios de estos cambios. El signo del número indica el efecto del factor sobre la desigualdad; por ejemplo, un número positivo indica un efecto desigualador y uno negativo indica igualador. Un número grande en valor absoluto indica la magnitud que tiene el factor sobre el cambio en la desigualdad con respecto a otros factores incluidos en la tabla, a su vez sugiere la importancia del efecto para ese factor. Por ejemplo, para el período para 1992-1996, el factor de los retornos a la educación fue el factor más importante para explicar los cambios en la desigualdad y dicho efecto fue desigualador.

En la primera columna de la tabla 5.1 tenemos los resultados promedio de la descomposición entre los años 1992 y 1996 con las encuestas ENH para los hogares urbanos. En la segunda columna se muestra a título ilustrativo la comparación entre los años 1996 y 2002 usando dos encuestas distintas. ${ }^{15}$ En las siguientes tres columnas se muestran los resultados para los períodos 2002-2005, 2005-2012 y 2002-2012, usando las bases de datos MESEP. Es importante notar que consistentemente con lo esperado, los

\footnotetext{
${ }^{14}$ El mecanismo se describe más detalladamente en la página 69 de Gasparini et al. (2005).

15 El ejercicio se realizado entre 1996 y 2002 es de forma exploratoria, dadas las restricciones de comparabilidad que se tienen con las encuestas ENH y MESEP.
} 
resultados entre 2002-2012 son aproximadamente iguales a la suma de las descomposiciones parciales entre 2002-2005 y 2005-2012.

Tabla 5.1. Descomposición de los cambios en el Coeficiente de Gini: Resultados promedio de los cambios en el año base en Colombia, períodos seleccionados

\begin{tabular}{|lrrrrr|}
\hline \multirow{2}{*}{ Indicador } & \multicolumn{5}{c|}{ Promedio } \\
\cline { 2 - 7 } & 1992-1996 & 1996-2002 & 2002-05 & 2005-2012 & 2002-2012 \\
\hline Observado & 4.41 & 2.07 & -1.46 & -1.58 & -3.04 \\
\hline Efecto & & & & & \\
1. Retornos a la educación & 0.93 & 0.72 & -0.88 & -0.07 & -0.92 \\
2. Brecha de género & 0.28 & 0.18 & -0.16 & -0.40 & -0.52 \\
3. Retornos a la experiencia & 0.00 & 0.82 & -0.18 & -0.31 & -0.40 \\
4. Factores no observados & 0.72 & 0.61 & -1.05 & -0.02 & -1.04 \\
5. Empleo & 0.42 & 0.51 & 0.13 & 0.53 & 0.66 \\
Horas de trabajo & 0.34 & -0.63 & 0.09 & 0.50 & 0.61 \\
Participación & 0.09 & 1.12 & 0.04 & 0.02 & 0.06 \\
6. Educación & 0.43 & 0.97 & 0.13 & -0.71 & -0.55 \\
\hline
\end{tabular}

Fuente: Cálculos propios basados en la ENH-Urbana y MESEP.

\section{Retornos a la educación}

Los resultados presentados en la Tabla 5.1 muestran que durante la década de los noventa los retornos a la educación fueron los más importantes en explicar el incremento en la desigualdad (0.93). Esto es coherente con los resultados del análisis no condicionado, dado que los individuos con los niveles educativos superiores fueron quienes obtuvieron mayores incrementos salariales entre 1992 y 1996. Los retornos continúan siendo desigualadores y con una importante magnitud de 0.72 entre 1996 y 2002.

La historia para la siguiente década muestra que este factor pasa a ser importante en contrarrestar la desigualdad para toda la década, con una importante magnitud entre 2002 y 2005, pero pierde relevancia entre 2005 y $2012 .{ }^{16}$ Este resultado puede ser explicado por los incrementos en los salarios reales para niveles educativos inferiores y la reducción de

\footnotetext{
${ }^{16}$ De hecho, para el período 2005-2012 cambia el signo de los retornos a la educación al analizar las descomposiciones originales alternando el período base (ver tabla A.5 del anexo), lo que implica algún tipo de inconsistencia, ya sea en la estrategia de descomposición o en problemas de comparabilidad en los datos, a pesar del esfuerzo de homogeneización de MESEP.
} 
los retornos a la educación para todos los niveles educativos, en mayor medida para los niveles educativos superiores. ${ }^{17}$

\section{Brecha de género}

Similar a los resultados de la sección 4.2, el parámetro de género de la ecuación de salarios muestra una leve reducción entre 1992-1996 y la brecha de género en los salarios promedio para hombres y mujeres de la tabla 4.4 se mantiene constante, el efecto de la brecha de género tuvo un efecto relativamente pequeño (0.28) y desigualador durante la década de los noventa. ${ }^{18}$

Para la década del 2000 el efecto de la brecha de género fue siempre igualador e importante (-0.52), especialmente en el período 2005-2012, a pesar de que el parámetro de la ecuación de salarios para género aumentó de manera importante entre 2002-2012. Para explicar este hecho, la tabla A.7 (del anexo) reporta el salario horario promedio por nivel educativo para hombres y mujeres, lo que permite establecer que la brecha de género es más pronunciada para el grupo de educación superior, pero los incrementos en los salarios han sido más importantes para las mujeres en los grupos educativos bajos. Las reducciones de la brecha son más relevantes en la parte inferior de la distribución que en la parte superior, lo que explica el efecto igualador durante la década. ${ }^{19}$

\footnotetext{
${ }^{17}$ Estos resultados son consistentes con las conclusiones de estudios como los de Vargas (2011) y Posso (2008) para Colombia, los cuales encuentran evidencia de disminuciones de los retornos a la educación para todos los niveles educativos, dada la convergencia para los salarios reales de los grupos educativos a excepción del nivel más alto, para el cual se incrementa la dispersión intragrupo para la década del 2000. Los autores atribuyen este hecho a factores institucionales para el caso de Vargas (2011) y cuestiones de calidad de la educación superior de Posso (2008).

${ }^{18}$ Tengamos en cuenta que para el período 1992-1996, en la tabla A.2 del anexo, el efecto es relativamente mayor y desigualador al tomar como período base 1992, el efecto cambia de dirección, siendo igualador si tomamos como período base 1996. Esto implica algún tipo de inconsistencia en la estrategia usada o en los datos.

${ }^{19}$ También puede explicarse por la evolución de los coeficientes del diferencial de género hallados por Vargas (2011), la cual encuentra evidencia sobre la forma de u invertida es un fenómeno reciente par el caso colombiano, esto es, a partir de 2003. Encuentra que la evolución de los coeficientes de la regresión por cuantiles del $(\log )$ de los salarios de las mujeres relativo al de los hombres en los percentiles 50 y 90 observaron una reducción importante en el diferencial para el período 1994-2006, mientras el diferencial para las mujeres en el percentil 10 no cambió significativamente, e incluso se incrementó a partir de 2004. Esto sugiere que sólo a partir de 2003 la evolución de los diferenciales para el percentil 50 son más bajos que aquellos para el percentil $10 \mathrm{y}$, por lo tanto, la forma de u invertida.
} 


\section{Retornos a la experiencia}

Los retornos a la experiencia resultan tener un efecto nulo sobre la desigualdad entre 1992 y $1996,{ }^{20}$ aunque parecen tener un fuerte efecto desigualador entre 1996 y 2002 (0.82). Al igual que lo observado en las estadísticas descriptivas, los incrementos salariales de la década del 2000 se dieron para los grupos de menor edad, lo que sugiere un efecto igualador que se respalda con los resultados de la Tabla 5.1, puesto que a lo largo de la década este efecto fue igualador, pero menos relevante en cuanto a magnitud (-0.4).

\section{Inobservables}

El efecto de los factores no observables fue fuerte durante la década de los noventa, debido al incremento en la dispersión de los inobservables en el año 1996 que tuvo una variación del $6.5 \%$ con respecto a 1992, por lo que fue el segundo en cuanto a magnitud entre 1992 y 1996 (0.72). En la siguiente década fue el más importante en contrarrestar la desigualdad (-1.04), debido en alguna medida a la reducción del desvío estándar de los residuos entre 2002 y 2012 del $-6.3 \% .^{21}$

\section{Empleo}

Para el período 1992 y 1996 tenemos un efecto horas de trabajo desigualador (0.42). Este hecho se explica probablemente por la reducción de las horas de trabajo para los niveles educativos inferiores (primaria incompleta y completa) entre 1992 y 1996 (tabla 4.5), pero que tuvo un mayor efecto en reducir las horas de trabajadas para los jefes de hogar de todos los niveles educativos (con excepción de superior incompleta) y que fue compensado por el incremento en la jornada laboral de las mujeres cónyuges para algunos niveles educativos (ver gráficos 4.2 y 4.3).

En este período el efecto participación es también desigualador, pero mucho menos importante (0.09). Pero debemos tener en cuenta que en este caso, los valores de las descomposiciones alternando el período base difieren de signo y tienen magnitudes

\footnotetext{
${ }^{20}$ Nótese que surge un problema similar que el comentario anterior; tomando como período base 1992 tiene un efecto desigualador importante, que es compensado exactamente en la misma magnitud si tomamos como período base 1996, pero esta vez igualador; lo que hace que en el promedio el efecto nulo (ver anexo A2).

${ }^{21}$ El efecto de los factores inobservables en el período 2005-2012 es pequeño, debido a una compensación al cambio de dirección cuando se toma como período base un año u el otro (ver tabla A5 del anexo). De todas formas ambos valores son de relativamente poca importancia. Tanto al analizar 2002-2005 (tabla A5) como al analizar 2002-2012 (tabla A6) las magnitudes y signos son consistentes con el valor promedio observado en la Tabla 5.1, lo que sugiere que el efecto es igualador y se concentra en la primera etapa.
} 
extremadamente altas (ver anexo A2). Esto implica que los resultados para este período en términos de participación (y consecuentemente de empleo) deben tomarse con cautela, dado que pueden ser sólo el reflejo de problemas de estimación y/o comparabilidad entre estas encuestas.

A lo largo de la segunda década, los efectos horas de trabajo y el efecto empleo son desigualadores (0.61 y 0.66 respectivamente); el efecto participación es también desigualador pero su magnitud es de sólo 0.06. Para el período 2002-2005, el efecto de las horas de trabajo y el efecto participación tienen un efecto pequeño en incrementar la desigualdad (0.09 y 0.04 respectivamente). Pero entre 2005 y 2012, el efecto horas es significativamente mayor en cuanto a magnitud (0.50), mientras que el efecto participación es muy reducido (0.02), por lo que en principio el efecto desigualador del mercado laboral para la década se explica principalmente por el efecto de las horas trabajadas durante el período 2005-2012. ${ }^{22}$

\section{Educación}

Los resultados sugieren un efecto desigualador importante de la estructura educativa durante la década de los noventa (0.43 en 1992-1996 y 0.97 en 1996-2002) que se explica por el efecto de los incrementos en los retornos a la educación de la década, a pesar de un leve cambio igualador en la estructura educativa. Para la década del 2000, inicialmente el efecto de la estructura educativa no tiene un peso muy relevante (0.13 para 2002-2005), pero para el período (2005-2012) el efecto es el mayor e igualador (-0.71). Este resultado es explicado por la reducción importante de los retornos a la educación durante la década cuyo efecto compensa el cambio desigualador en la estructura educativa durante el mismo período como se menciona en la sección 4.7.

\section{DESCOMPOSICIÓN DE INGRESOS TOTALES POR FUENTE DE INGRESOS}

El análisis mediante descomposiciones microeconométricas de la sección anterior se restringe a los determinantes de los ingresos laborales, por varias razones: las encuestas de

\footnotetext{
${ }^{22}$ Es importante notar que entre 2005 y 2012 se presentó una leve disminución de las horas de trabajo en niveles educativos bajos (ver tabla 4.5), y particularmente una disminución generalizada de las horas de trabajo para las mujeres en todos los grupos educativos (con excepción de superior incompleta), al tiempo que los hombres incrementan sus horas en $0.2 \%$.
} 
hogares de Colombia presentan limitaciones en capturar otras variables que permitan modelar otras fuentes de ingresos como los ingresos no laborales, la renta implícita o las transferencias estatales; ${ }^{23}$ también es la razón principal por la que se dispone de abundante literatura sobre aplicaciones metodológicas con los ingresos laborales para países de Latinoamérica.

Un elemento adicional de análisis que complementa los resultados de los ejercicios anteriores es la descomposición del índice de Gini por fuente de ingresos; de esta manera podemos evaluar la tendencia y la contribución de cada una de las fuentes de ingreso en la desigualdad.

\subsection{Metodología}

En la descomposición del índice de Gini por fuente de ingreso, se divide el ingreso per cápita familiar de cada persona en los montos provenientes de sus componentes: ingreso laboral, ingreso no laboral y renta implícita de la vivienda propia. Siguiendo a Gasparini et al. (2013) el Gini puede escribirse en función de la covarianza del ingreso y su rango, $G(x)=2 \operatorname{cov}(x, F(x)) / \mu$; a partir de allí Lerman y Yitzhaki (1985) derivan la siguiente expresión:

$$
G(x)=\sum_{k=1}^{K}\left[\frac{\operatorname{cov}\left(x_{k}, F(x)\right)}{\operatorname{cov}\left(x_{k}, F\left(x_{k}\right)\right)}\right]\left[\frac{\operatorname{cov}\left(x_{k}, F\left(x_{k}\right)\right)}{\mu_{k}}\right] \frac{\mu_{k}}{\mu}
$$

que se resume a $G(x)=\sum_{k=1}^{K} R_{k} G\left(x_{k}\right) s_{k}$.

Donde $G\left(x_{k}\right)$ es el Gini del ingreso de la fuente $k, s_{k}$ es la participación de la fuente k en el ingreso total y $R_{k}$ es la correlación-Gini entre el ingreso de la fuente $k$ y el ingreso total.

\subsection{Resultados}

Las tablas 6.1 a las 6.3 reportan los resultados de varios ejercicios de la descomposición de la desigualdad del ingreso per cápita familiar (ipcf) por fuentes de

\footnotetext{
23 Gasparini et al. (2013) señalan que en general las encuestas de hogares latinoamericanas captan esencialmente ingresos laborales y transferencias, dado que en promedio el $75 \%$ de los ingresos familiares en dichas encuestas provienen de fuentes laborales.
} 
ingreso. ${ }^{24}$ La tabla 6.1 comprende el período 1992-2012; para incluir consistentemente el primer año se construye el ipcf como la suma de los ingresos laborales e ingresos no laborales y el ejercicio se realiza sólo para zonas urbanas. La tabla 6.2 comprende el período 1996-2012 para el total nacional. En esta tabla se construye el ipcf como la suma del ingreso laboral, ingreso no laboral y la renta implícita de la vivienda propia, utilizando un método simple y estándar para esta imputación de la renta en todas las encuestas (incluyendo las de Mesep). ${ }^{25}$ La tabla 6.3 realiza el mismo ejercicio de descomposición anterior, pero usando la renta imputada de la metodología Mesep, sólo para las bases del período 2002-2012.

Tabla 6.1. Descomposición del Gini por Fuente de Ingreso para Colombia para hogares urbanos. Ingresos laborales e ingresos no laborales

\begin{tabular}{|c|c|cc|cc|cc|cc|}
\hline \multirow{2}{*}{ Año } & \multirow{2}{*}{ Gini } & \multicolumn{2}{|c|}{$\begin{array}{c}\text { Participación en el } \\
\text { ingreso total (Sk) }\end{array}$} & \multicolumn{2}{|c|}{$\begin{array}{c}\text { Gini por fuente de } \\
\text { ingreso (Gk) }\end{array}$} & \multicolumn{2}{|c|}{$\begin{array}{c}\text { Correlación con el } \\
\text { ingreso total (Rk) }\end{array}$} & \multicolumn{2}{|c|}{$\begin{array}{c}\text { Contribución por } \\
\text { fuente }\end{array}$} \\
\cline { 3 - 10 } & & ilapcf & inlapcf & ilapcf & inlapcf & ilapcf & inlapcf & ilapcf & inlapcf \\
\hline 1992 & 47.48 & 84.40 & 15.60 & 49.93 & 87.83 & 0.918 & 0.642 & 38.67 & 8.80 \\
1996 & 51.59 & 84.69 & 15.31 & 54.15 & 87.60 & 0.934 & 0.653 & 42.83 & 8.76 \\
2000 & 54.52 & 80.64 & 19.36 & 58.48 & 86.51 & 0.912 & 0.688 & 43.01 & 11.52 \\
2002 & 56.11 & 80.97 & 19.03 & 58.16 & 88.17 & 0.928 & 0.741 & 43.70 & 12.43 \\
2003 & 54.52 & 82.26 & 17.74 & 56.54 & 88.37 & 0.926 & 0.731 & 43.07 & 11.46 \\
2004 & 55.18 & 81.51 & 18.49 & 57.07 & 88.63 & 0.926 & 0.739 & 43.08 & 12.11 \\
2005 & 54.48 & 80.80 & 19.20 & 56.48 & 87.78 & 0.920 & 0.741 & 41.98 & 12.49 \\
2008 & 54.96 & 80.16 & 19.84 & 57.22 & 87.58 & 0.920 & 0.735 & 42.20 & 12.77 \\
2010 & 54.29 & 79.91 & 20.09 & 56.95 & 85.95 & 0.920 & 0.719 & 41.87 & 12.42 \\
2011 & 53.21 & 80.06 & 19.94 & 55.93 & 85.07 & 0.920 & 0.707 & 41.20 & 11.99 \\
2012 & 52.03 & 80.80 & 19.20 & 54.90 & 84.62 & 0.921 & 0.689 & 40.85 & 11.19 \\
\hline
\end{tabular}

Nota: ilapcf: ingreso laboral per cápita familiar; inlapcf: ingreso no laboral per cápita familiar.

Fuente: Cálculos propios basados en MESEP.

\footnotetext{
${ }^{24}$ En la tabla 6.3 la construcción de la serie de ingreso per cápita familiar no incluye un empalme que se adiciona en la metodología Mesep, por lo cual, los niveles de índice de Gini del ipcf no coinciden con los valores de las tablas A4, A5, A6 del Anexo 1, para los años 2002, 2005 y 2012.

${ }^{25}$ En este caso la renta imputada por propiedad de la vivienda consiste en imputarle a los hogares propietarios de la vivienda un 10\% del ingreso total familiar. Este método fue aplicado en la ENH de 1996 en los ejercicios de descomposiciones microeconométricas.
} 
Tabla 6.2. Descomposición del Gini por fuente de ingreso para Colombia. Renta imputada con metodología estándar

\begin{tabular}{|c|c|c|c|c|c|c|c|c|c|c|c|c|c|}
\hline \multirow{2}{*}{ Año } & \multirow{2}{*}{ Gini } & \multicolumn{3}{|c|}{$\begin{array}{c}\text { Participación en el } \\
\text { ingreso total (Sk) }\end{array}$} & \multicolumn{3}{|c|}{$\begin{array}{c}\text { Gini por fuente de } \\
\text { ingreso (Gk) }\end{array}$} & \multicolumn{3}{|c|}{$\begin{array}{l}\text { Correlación con el } \\
\text { ingreso total (Rk) }\end{array}$} & \multicolumn{3}{|c|}{$\begin{array}{l}\text { Contribución por } \\
\text { fuente }\end{array}$} \\
\hline & & ilapcf & inlapcf & $\begin{array}{l}\text { Renta } \\
\text { impcf }\end{array}$ & ilapcf & inlapcf & $\begin{array}{l}\text { Renta } \\
\text { impcf }\end{array}$ & ilapcf & inlapcf & $\begin{array}{l}\text { Renta } \\
\text { impcf }\end{array}$ & ilapcf & inlapcf & $\begin{array}{l}\text { Renta } \\
\text { impcf }\end{array}$ \\
\hline 1996 & 54.93 & 80.09 & 13.54 & 6.37 & 56.67 & 90.00 & 71.43 & 0.940 & 0.702 & 0.813 & 42.66 & 8.55 & 3.70 \\
\hline 2000 & 57.51 & 77.68 & 16.33 & 5.99 & 60.04 & 89.56 & 74.41 & 0.926 & 0.732 & 0.812 & 43.19 & 10.71 & 3.62 \\
\hline 2002 & 58.37 & 76.98 & 17.31 & 5.71 & 59.58 & 89.96 & 76.04 & 0.934 & 0.772 & 0.803 & 42.84 & 12.02 & 3.49 \\
\hline 2003 & 56.33 & 78.63 & 15.81 & 5.56 & 57.33 & 90.35 & 75.50 & 0.934 & 0.765 & 0.784 & 42.10 & 10.93 & 3.29 \\
\hline 2004 & 57.25 & 77.63 & 16.73 & 5.64 & 58.16 & 90.51 & 76.04 & 0.934 & 0.772 & 0.794 & 42.17 & 69 & 1 \\
\hline 2005 & 56.64 & 76.73 & 17.60 & 5.68 & 57.84 & 89.27 & 75.24 & 0.929 & 0.767 & 0.790 & 41.23 & 12.05 & 3.38 \\
\hline 2008 & 57.83 & 76.68 & 17.94 & 5.38 & 59.02 & 89.43 & 79.88 & 0.928 & 0.770 & 0.808 & 42.00 & 12.35 & 3.47 \\
\hline 2010 & 56.95 & 76.30 & 18.36 & 5.35 & 58.58 & 87.55 & 79.83 & 0.928 & 0.749 & 0.806 & 41.48 & 12.04 & 3.44 \\
\hline 2011 & 55.91 & 76.48 & 18.26 & 5.26 & 57.61 & 86.66 & 79.50 & 0.928 & 0.739 & 0.796 & 40.89 & 11.69 & 3.33 \\
\hline 2012 & 54.92 & 77.18 & 17.70 & 5.11 & 56.78 & 86.21 & 80.09 & 0.928 & 0.722 & 0.789 & 40.67 & 11.02 & 3.23 \\
\hline
\end{tabular}

Nota: ilapcf: ingreso laboral per cápita familiar; inlapcf: ingreso no laboral per cápita familiar; ripcf: renta implícita de la vivienda propia per cápita familiar Fuente: Cálculos propios basados en MESEP.

Tabla 6.3. Descomposición del Gini por fuente de ingreso para Colombia. Renta imputada con metodología MESEP.

\begin{tabular}{|c|c|c|c|c|c|c|c|c|c|c|c|c|c|}
\hline \multirow{2}{*}{ Año } & \multirow{2}{*}{ Gini } & \multicolumn{3}{|c|}{$\begin{array}{l}\text { Participación en el } \\
\text { ingreso total (Sk) }\end{array}$} & \multicolumn{3}{|c|}{$\begin{array}{l}\text { Gini por fuente de } \\
\text { ingreso (Gk) }\end{array}$} & \multicolumn{3}{|c|}{$\begin{array}{l}\text { Correlación con el } \\
\text { ingreso total (Rk) }\end{array}$} & \multicolumn{3}{|c|}{$\begin{array}{l}\text { Contribución por } \\
\text { fuente }\end{array}$} \\
\hline & & ilapcf & nlapcf & $\begin{array}{l}\text { Renta } \\
\text { impcf }\end{array}$ & ilapcf & nlapc & $\begin{array}{l}\text { Renta } \\
\text { impcf }\end{array}$ & ilapcf & inlapcf & $\begin{array}{l}\text { Renta } \\
\text { impcf }\end{array}$ & ilapcf & inlapcf & $\begin{array}{l}\text { Renta } \\
\text { impcf }\end{array}$ \\
\hline 2002 & 56.05 & 72.87 & 16.39 & 10.74 & 59.88 & 90.04 & 70.21 & 0.921 & 0.778 & 0.585 & 40.17 & 11.48 & 4.41 \\
\hline 2003 & 54.28 & 74.65 & 15.01 & 10.34 & 57.68 & 90.43 & 70.57 & 0.920 & 0.772 & 0.573 & 39.62 & 10.48 & 4.18 \\
\hline 2004 & 54.84 & 73.41 & 15.82 & 10.76 & 58.33 & 90.55 & 69.90 & 0.919 & 0.777 & 0.576 & 39.37 & 11.12 & 4.33 \\
\hline 2005 & 54.78 & 73.22 & 16.79 & 9.99 & 57.99 & 89.31 & 71.33 & 0.917 & 0.772 & 0.602 & 38.92 & 11.57 & 4.29 \\
\hline 2008 & 56.65 & 73.09 & 17.10 & 9.81 & 59.39 & 89.53 & 74.67 & 0.916 & 0.784 & 0.666 & 39.78 & 12.00 & 4.88 \\
\hline 2010 & 55.70 & 73.14 & 17.60 & 9.26 & 58.74 & 87.59 & 75.13 & 0.916 & 0.763 & 0.661 & 39.34 & 11.77 & 4.60 \\
\hline 2011 & 54.55 & 73.38 & 17.51 & 9.11 & 57.73 & 86.69 & 75.03 & 0.915 & 0.753 & 0.642 & 38.74 & 11.42 & 4.39 \\
\hline 2012 & 53.63 & 73.98 & 16.97 & 9.06 & 56.91 & 86.25 & 75.67 & 0.914 & 0.737 & 0.635 & 38.48 & 10.79 & 4.35 \\
\hline
\end{tabular}

Nota: ilapcf: ingreso laboral per cápita familiar; inlapcf: ingreso no laboral per cápita familiar; ripcf: renta implícita de la vivienda propia per cápita familiar Fuente: Cálculos propios basados en MESEP.

Para cada una de las tablas 6.1 a 6.3, la primera columna presenta el coeficiente de Gini del ipcf. El siguiente grupo de columnas reporta la participación de cada una de las fuentes de ingreso en el ingreso total, siendo los ingresos laborales los de mayor participación, seguido por los ingresos no laborales y renta implícita. Las columnas del siguiente grupo presentan los coeficientes de Gini por fuente de ingreso. Se observa que los 
ingresos no laborales presentan una mayor concentración del ingreso, seguido de los ingresos laborales. Sin embargo la correlación con el ingreso total (que se muestra en el tercer grupo de columnas) es mucho mayor para los ingresos laborales, con niveles por encima 0.91 para todo el período, lo que sugiere que los ingresos laborales presentan un efecto más importante en la distribución del ingreso total. Esto se evidencia en el último grupo de columnas donde se computa la contribución parcial al coeficiente de Gini, de cada una de las fuentes. ${ }^{26}$

La contribución parcial en el coeficiente de Gini de los ingresos laborales (para cada una de las metodologías) muestra una marcada tendencia hacia la disminución, comparado con los ingresos no laborales, que también disminuyen su contribución, pero en una menor medida; de hecho presentan una evolución cóncava, en la cual se incrementan entre 20022008 y luego disminuyen levemente entre 2010-2012. En cuanto a la contribución parcial de la renta imputada, esta no presenta un patrón claro, con un importante incremento en 2008. En cuanto a nivel, la contribución parcial de la renta imputada con la metodología Mesep presenta una diferencia de un punto con respecta a la metodología estándar para ese período.

En cuanto a la renta imputada se presentan algunas diferencias importantes entre la metodología estándar y la Mesep. En términos de nivel, el Gini de la renta imputada es mucho mayor con la metodología estándar que con la Mesep, teniendo un nivel promedio de 76.8 con la primera y de 72.8 con la segunda, para el período 2002-2012. También la correlación es mucho mayor con la metodología estándar que con la Mesep, siendo en promedio para el período de 0.80 y 0.62 respectivamente. Este hecho explica las diferencias en el índice de Gini del ingreso total, el cual es en promedio 1.6 puntos del Gini mayor con la metodología estándar, lo que implica un efecto igualador de la metodología de la renta imputada de la Mesep cuando se adiciona al ingreso total.

De todas formas, en términos de evolución de la renta implícita, ambas metodologías presentan patrones similares: desde 2002 la participación tiende a caer y la desigualdad tiende a aumentar, su correlación con el ingreso total cae entre 2002-2003, aumenta en el tramo 2003-2008 para luego presentar una tendencia decreciente. Adicionalmente en la tabla 6.2 podemos ver que entre 1996 y 2002 la desigualdad aumentó, pero la participación

\footnotetext{
${ }^{26}$ Es decir, $R_{k} G\left(x_{k}\right) s_{k}$ para cada la fuente $k$.
} 
y la correlación fueron decrecientes durante ese lapso, lo que provocó una reducción de su contribución a la desigualdad total.

La confección de la tabla 6.2 nos ilustra por qué la descomposición entre 1996 y 2002 es sólo ilustrativa; el cambio en el Gini de la Tabla 5.1 es de 2.07, mientras que al utilizar (en la tabla 6.2) la misma metodología de imputación de la renta, el incremento pasa a ser de 3.44 puntos. $^{27}$

En síntesis, se observa que los ingresos laborales son los más importantes en explicar los cambios en la desigualdad durante las últimas dos décadas, pero éstos pierden alguna relevancia en cuanto a su participación y desigualdad, manteniendo constante su correlación. Por su parte, los ingresos no laborales ganan relevancia en cuanto a participación, pasando del 16.39 en 2002 al 16.97 en 2012, pero disminuyen marginalmente su desigualdad y correlación (ver tabla 6.3). Por último, la renta imputada presenta diferencias importantes en términos de nivel al utilizar metodologías alternativas pero su evolución es similar en cuanto a su participación, desigualdad y correlación con el ingreso total.

\section{COMENTARIOS FINALES}

Este trabajo contribuye a explicar el fenómeno del incremento de la desigualdad de ingresos en Colombia desde la década de los noventa. Para ello propone una metodología alternativa a las técnicas de descomposición aplicadas en trabajos anteriores. En particular la aplicada por Gasparini et al. (2005) y otros autores en varios países de Latinoamérica.

Los resultados de este estudio sugieren que para la década de los noventa, en general los determinantes del ingreso laboral fueron desigualadores; los factores más importantes en explicar el incremento en la desigualdad fueron los retornos a la educación, seguido de los factores no observados y la estructura educativa. Por su parte, la brecha de género y los retornos a la experiencia son factores que tienen poca importancia en explicar el incremento de la desigualdad durante el período.

\footnotetext{
${ }^{27}$ Importante notar que en la Tabla 5.1 el ejercicio 1996-2002 se hizo con la Mesep 2002 para el III trimestre, para aumentar la comparablilidad con la base ENH 1996, además que la renta imputada en 2002 para ese mismo ejercicio es de la metodología Mesep que, como se explicó, genera un efecto igualador en el coeficiente Gini del ipcf.
} 
Para la década del 2000, tenemos claros dos conjuntos de factores que van en direcciones opuestas. Por un lado tenemos el conjunto de los retornos a las características observables, los factores no observados y la estructura educativa, que en mayor o menor medida tienen un efecto igualador durante la década. Por otro lado, tenemos los efectos del mercado laboral (principalmente las horas de trabajo) presentan un efecto desigualador importante durante este período.

Buena parte de los cambios en la desigualdad en Colombia durante estas dos décadas se explican por los cambios en la distribución de los ingresos laborales, pero la evidencia de la sección 6 sugiere que las otras fuentes de ingresos muestran dinámicas que afectan a la evolución de la desigualdad. En la medida que la GEIH mantenga su comparabilidad, será interesante evaluar en el futuro si las tendencias persisten, así como examinar los determinantes de estas fuentes de ingreso y su contribución en la desigualdad.

La metodología de descomposiciones microeconométricas es perfecta para el estudio de los problemas de distribución del ingreso, debido a su versatilidad y flexibilidad; ésta se puede ajustar bien sea a los objetivos particulares del investigador, o a las carencias de los tipos datos ofrecidos por las Encuestas de Hogares de Latinoamérica (como es el caso de los datos longitudinales). Al modelo básico se le pueden ir agregando más componentes como son la modelación de la informalidad, los retornos regionales, las decisiones de fertilidad o escolaridad; cada una de estas con sus respectivas estrategias de estimación que sería un significativo aporte a la literatura colombiana sobre temas distributivos. 


\section{BIBLIOGRAFÍA}

Arango, L., Posada, C., y Uribe, J. (2004), "Cambios en la Estructura de los Salarios Urbanos en Colombia (1984-2000)", Borradores de Economía No. 297, Banco de la República.

Attanasio, O., Goldberg, P., y Pavcnik, N. (2004), "Trade Reforms and Wage Inequality in Colombia", Journal of development Economics, 74 (2): 331-366.

Autor, D., Katz, L., y Krueger, A. (1998), “Computing Inequality: Have Computers Changed the Labor Market?", Quarterly Journal of Economics, 113 (4):1169-1214.

Bernal, R., Cárdenas, M., Núñez, J., y Sánchez, F. (1997), "Macroeconomic performance and inequality in Colombia: 1976-1996", Documento de trabajo No. 1, Fedesarrollo.

Bernal, R., y Cárdenas, M. (1999), "Changes in the distribution of income and the new economic model in Colombia”, Serie Reformas Económicas No. 36, CEPAL.

Blinder, A. (1973), "Wage Discrimination: Reduced Form and Structural Estimate", The Journal of Human Resources, 8 (4): 436-453.

Bourguignon, F., Ferreira, F. y Lustig, N. (1998) "The microeconomics of income distribution dynamics in East Asia and Latin America", IDB-World Bank Research Proposal.

Galvis, Luis A. (2007), "La topografía económica de Colombia”, en Adolfo Meisel Roca (ed), Regiones, ciudades y crecimiento económico en Colombia, Bogotá, Colección de Economía Regional, Banco de la República.

Gasparini, L., Marchionni, M. y Sosa, W. (2005), "Characterization of Inequality Changes through Microeconometric Decompositions. The Case of Greater Buenos Aires", Capitulo 2, p. 47-81, en Bourguignon, F., Ferreira, F. y N. Lustig, (eds). The Microeconomics of Income Distribution Dynamics in East Asia and Latin America. Washington, DC: The World Bank/ Oxford University Press.

Gasparini, L., Cicowiez, M., y Sosa, W. (2013). Pobreza y Desigualdad en América Latina: Conceptos, herramientas y aplicaciones. Buenos Aires: Temas. 
Haimovich, F., Winkler, H. y Gasparini, L. (2006), "Distribución del Ingreso en América Latina: Explorando las Diferencias entre Países”, Documento de Trabajo No. 34, Centro de Estudios Distributivos, Laborales y Sociales.

Heckman, J. (1974), "Shadow prices, market wages, and labor supply”, Econometrica, 42 (4): 679-694.

Katz, L,. y Murphy, K. (1992), “Changes in relative wages, 1963 - 1987: supply and demand factors", The Quarterly Journal of Economics, 107(1):35-78.

Lerman y Yitzhaki (1985), "Income inequality effects by income source: a new approach and application to the U.S.”, Review of Economics and Statistics 67 (1): 151-156.

Melly, B. (2005), "Descomposition of Differences in Distribution Using Quantile Regression", Journal of Labour Economics, 12(4): 577-590.

Mincer, J. (1974). Schooling, experience and earnings. New York: Columbia University Press.

Núñez, J. y Sánchez, F. (1998), “Descomposición de la Desigualdad del Ingreso Laboral Urbano en Colombia: 1976-1997”, Archivos de Macroeconomía No. 86, Unidad de Análisis Macroeconómico - Departamento Nacional de Planeación.

Posso, C. (2008), "Desigualdad Salarial en Colombia 1984-2005: Cambios en la Composición del Mercado Laboral y Retornos a la Educación Post-Secundaria", Borradores de Economía No. 529, Banco de la República.

Shorrocks, A. (1982), "Inequality decomposition by factor components", Econometrica, 50 (1): 193-211.

Vargas, C. O. (2011), "Desigualdad de salarios en Colombia: evidencia a partir de encuestas de hogares 1984-2010”, Borradores de Economía No. 661, Banco de la República de Colombia.

Zoloa, J. (2011), “Los Cambios en la Distribución del Ingreso de Argentina entre 1998 y 2005", Documento de Trabajo No. 122, Centro de Estudios Distributivos, Laborales y Sociales. 


\section{ANEXO}

\begin{tabular}{|c|c|}
\hline \multicolumn{2}{|r|}{ Cuadro A.1. Descripción de variables } \\
\hline Variable & Descripción \\
\hline $\begin{array}{l}\text { Población Económicamente } \\
\text { Activa }\end{array}$ & $\begin{array}{l}\text { Individuos entre } 12 \text { y } 65 \text { años con respuestas coherentes y que no perciben } \\
\text { ingresos por jubilación }\end{array}$ \\
\hline \multicolumn{2}{|l|}{ Ingresos } \\
\hline Salario horario & Ingreso laboral horario \\
\hline \multicolumn{2}{|l|}{ Rol en el hogar } \\
\hline $\begin{array}{l}\text { Jefe } \\
\text { Cónyuge } \\
\text { Otros miembros del hogar }\end{array}$ & $\begin{array}{l}1 \text { si el individuo declara ser jefe del hogar. } 0 \text { en caso contrario } \\
1 \text { si el individuo declara ser cónyuge. } 0 \text { en caso contrario } \\
1 \text { si el individuo no declara ser ni jefe ni cónyuge. } 0 \text { en caso contrario }\end{array}$ \\
\hline \multicolumn{2}{|l|}{ Características generales } \\
\hline $\begin{array}{l}\text { Hombre } \\
\text { Edad } \\
\text { Menor de } 18 \text { años } \\
\text { Casado } \\
\text { Hijos menores } \\
\end{array}$ & $\begin{array}{l}1 \text { si el individuo es de sexo masculino. } 0 \text { en caso contrario } \\
\text { Edad en años } \\
1 \text { si el individuo declara tener menos de } 18 \text { años de edad. } 0 \text { en caso contrario } \\
1 \text { si el individuo declara estar casado o unido. } 0 \text { en caso contrario } \\
\text { Número de hijos menores de } 18 \text { años que habitan en el hogar. }\end{array}$ \\
\hline \multicolumn{2}{|l|}{ Características Educativas } \\
\hline $\begin{array}{l}\text { Primaria incompleta } \\
\text { Primaria completa } \\
\text { Secundaria incompleta } \\
\text { Secundaria completa } \\
\text { Superior incompleta } \\
\text { Superior completa } \\
\text { Asiste }\end{array}$ & $\begin{array}{l}1 \text { si el individuo declara tener primaria incompleta o menos. } 0 \text { en caso contrario } \\
1 \text { si el individuo declara tener primaria completa. } 0 \text { en caso contrario } \\
1 \text { si el individuo declara tener secundaria incompleta. } 0 \text { en caso contrario } \\
1 \text { si el individuo declara tener secundaria completa. } 0 \text { en caso contrario } \\
1 \text { si el individuo declara tener superior incompleto. } 0 \text { en caso contrario } \\
1 \text { si el individuo declara tener superior completo o más. } 0 \text { en caso contrario } \\
1 \text { si el individuo declara asistir a algún centro educativo en cualquier nivel. } 0 \text { en } \\
\text { caso contrario }\end{array}$ \\
\hline \multicolumn{2}{|l|}{ Características Laborales } \\
\hline $\begin{array}{l}\text { Horas trabajadas } \\
\text { Ocupado } \\
\text { Desocupado } \\
\text { Inactivo } \\
\text { Jefe Ocupado } \\
\text { Cónyuge ocupado }\end{array}$ & $\begin{array}{l}\text { Horas trabajadas semanales en todos los empleos (ocupación principal y } \\
\text { secundaria) } \\
1 \text { si el individuo declara estar ocupado. } 0 \text { en caso contrario } \\
1 \text { si el individuo declara estar desocupado. } 0 \text { en caso contrario } \\
1 \text { si el individuo declara estar inactivo. } 0 \text { en caso contrario } \\
1 \text { si el jefe del hogar está ocupado. } 0 \text { en caso contrario } \\
1 \text { si el cónyuge está ocupado. } 0 \text { en caso contrario } \\
\end{array}$ \\
\hline \multicolumn{2}{|l|}{ Características espaciales } \\
\hline $\begin{array}{l}\text { Urbano } \\
\text { Región } 01 \\
\text { Región } 02 \\
\text { Región } 03 \\
\text { Región } 04 \\
\text { Región } 05 \\
\end{array}$ & $\begin{array}{l}1 \text { si el individuo se encuentra en una zona urbana. } 0 \text { en caso contrario } \\
\text { Chocó } \\
\text { Bogotá } \\
\text { Antioquia, Boyacá, Caldas, Cundinamarca, Quindío, Risaralda, Santander, } \\
\text { Tolima y Valle del Cauca } \\
\text { Atlántico, Bolívar, Cesar, Córdoba, Guajira, Magdalena, Norte de Santander y } \\
\text { Sucre. } \\
\text { Cauca, Huila, Nariño, Caquetá y Meta. }\end{array}$ \\
\hline
\end{tabular}


Tabla A.2. Descomposición de los Cambios en el Coeficiente de Gini: Ingreso Laborales en Colombia, ENH 1992 - ENH 1996

\begin{tabular}{|c|c|c|c|c|c|}
\hline \multicolumn{3}{|l|}{ Período base 1992} & \multicolumn{3}{|l|}{ Período base 1996} \\
\hline \multirow{2}{*}{ Indicador } & \multicolumn{2}{|c|}{ Ingresos } & \multirow{2}{*}{ Indicador } & \multicolumn{2}{|c|}{ Ingresos } \\
\hline & Nivel & Cambio & & Nivel & Cambio \\
\hline 1992 observado & 47.47 & & 1992 observado & 47.47 & 4.41 \\
\hline 1996 observado & 51.88 & 4.41 & 1996 observado & 51.88 & \\
\hline Efecto & & & Efecto & & \\
\hline 1. Retornos a la educación & 49.07 & 1.60 & 1. Retornos a la educación & 51.62 & 0.26 \\
\hline 2. Brecha de género & 48.33 & 0.86 & 2. Brecha de género & 52.19 & -0.31 \\
\hline 3. Retornos a la experiencia & 48.06 & 0.59 & 3. Retornos a la experiencia & 52.48 & -0.60 \\
\hline 4. Factores no observados & 48.76 & 1.29 & 4. Factores no observados & 51.74 & 0.14 \\
\hline 5. Empleo & 50.27 & 2.80 & 5. Empleo & 53.85 & -1.97 \\
\hline Horas de trabajo & 47.85 & 0.38 & Horas de trabajo & 51.59 & 0.29 \\
\hline Participación & 49.91 & 2.44 & Participación & 54.13 & -2.25 \\
\hline 6. Educación & 48.02 & 0.55 & 6. Educación & 51.57 & 0.31 \\
\hline
\end{tabular}

Fuente: Cálculos propios basados en la ENH-FT III trimestre (urbano).

Tabla A.3. Descomposición de los Cambios en el Coeficiente de Gini: Ingreso Laborales en Colombia, ENH 1996 - MESEP 2002

\begin{tabular}{|c|c|c|c|c|c|}
\hline \multicolumn{3}{|l|}{ Período base 1996} & \multicolumn{3}{|l|}{ Período base 2002} \\
\hline \multirow{2}{*}{ Indicador } & \multicolumn{2}{|c|}{ Ingresos } & \multirow{2}{*}{ Indicador } & \multicolumn{2}{|c|}{ Ingresos } \\
\hline & Nivel & Cambio & & Nivel & Cambio \\
\hline 1996 observado & 54.93 & & 1996 observado & 54.93 & 2.07 \\
\hline 2002 observado & 57.00 & 2.07 & 2002 observado & 57.00 & \\
\hline Efecto & & & Efecto & & \\
\hline 1. Retornos a la educación & 55.37 & 0.44 & 1. Retornos a la educación & 56.00 & 1.00 \\
\hline 2. Brecha de género & 55.01 & 0.08 & 2. Brecha de género & 56.72 & 0.28 \\
\hline 3. Retornos a la experiencia & 56.39 & 1.46 & 3. Retornos a la experiencia & 56.81 & 0.19 \\
\hline 4. Factores no observados & 55.50 & 0.57 & 4. Factores no observados & 56.35 & 0.65 \\
\hline 5. Empleo & 56.23 & 1.30 & 5. Empleo & 56.27 & -0.27 \\
\hline Horas de trabajo & 53.88 & -1.05 & Horas de trabajo & 56.22 & -0.22 \\
\hline Participación & 56.22 & 2.29 & Participación & 57.05 & -0.05 \\
\hline 6. Educación & 55.65 & 0.72 & 6. Educación & 55.78 & 1.22 \\
\hline
\end{tabular}

Fuente: Cálculos propios basados en la ENH-FT y MESEP.

Nota: la ENH-FT de 1996 es nacional se usan datos urbano-rurales y para la MESEP se usan solo los datos del III trimestre de 2002, para hacer aproximados los tamaños de ambas muestras. 
Tabla A.4. Descomposición de los Cambios en el Coeficiente de Gini: Ingreso Laborales en Colombia, MESEP 2002 - MESEP 2005

\begin{tabular}{|c|c|c|c|c|c|}
\hline \multicolumn{3}{|l|}{ Período base 2002} & \multicolumn{3}{|l|}{ Período base 2005} \\
\hline \multirow{2}{*}{ Indicador } & \multicolumn{2}{|c|}{ Ingresos } & \multirow{2}{*}{ Indicador } & \multicolumn{2}{|c|}{ Ingresos } \\
\hline & Nivel & Cambio & & Nivel & Cambio \\
\hline 2002 observado & 56.56 & & 2002 observado & 56.56 & -1.46 \\
\hline 2005 observado & 55.10 & -1.46 & 2005 observado & 55.10 & \\
\hline Efecto & & & Efecto & & \\
\hline 1. Retornos a la educación & 55.68 & -0.88 & 1. Retornos a la educación & 55.97 & -0.87 \\
\hline 2. Brecha de género & 56.40 & -0.16 & 2. Brecha de género & 55.25 & -0.15 \\
\hline 3. Retornos a la experiencia & 56.34 & -0.22 & 3. Retornos a la experiencia & 55.25 & -0.15 \\
\hline 4. Factores no observados & 55.57 & -0.99 & 4. Factores no observados & 56.20 & -1.10 \\
\hline 5. Empleo & 56.80 & 0.24 & 5. Empleo & 55.08 & 0.02 \\
\hline Horas de trabajo & 56.69 & 0.13 & Horas de trabajo & 55.05 & 0.05 \\
\hline Participación & 56.67 & 0.11 & Participación & 55.13 & -0.03 \\
\hline 6. Educación & 56.53 & -0.03 & 6. Educación & 54.81 & 0.29 \\
\hline
\end{tabular}

Fuente: Cálculos propios basados en la MESEP.

Tabla A.5. Descomposición de los Cambios en el Coeficiente de Gini: Ingreso Laborales en Colombia, MESEP 2005 - MESEP 2012

\begin{tabular}{|c|c|c|c|c|c|}
\hline \multicolumn{3}{|l|}{ Período base 2005} & \multicolumn{3}{|l|}{ Período base 2012} \\
\hline \multirow{2}{*}{ Indicador } & \multicolumn{2}{|c|}{ Ingresos } & \multirow{2}{*}{ Indicador } & \multicolumn{2}{|c|}{ Ingresos } \\
\hline & Nivel & Cambio & & Nivel & Cambio \\
\hline 2005 observado & 55.10 & & 2005 observado & 55.10 & -1.58 \\
\hline 2012 observado & 53.52 & -1.58 & 2012 observado & 53.52 & \\
\hline Efecto & & & Efecto & & \\
\hline 1. Retornos a la educación & 55.35 & 0.25 & 1. Retornos a la educación & 53.91 & -0.39 \\
\hline 2. Brecha de género & 55.03 & -0.07 & 2. Brecha de género & 54.24 & -0.72 \\
\hline 3. Retornos a la experiencia & 55.24 & 0.14 & 3. Retornos a la experiencia & 54.28 & -0.76 \\
\hline 4. Factores no observados & 55.39 & 0.29 & 4. Factores no observados & 53.86 & -0.34 \\
\hline 5. Empleo & 55.74 & 0.64 & 5. Empleo & 53.11 & 0.41 \\
\hline Horas de trabajo & 55.68 & 0.58 & Horas de trabajo & 53.10 & 0.42 \\
\hline Participación & 55.16 & 0.06 & Participación & 53.54 & -0.02 \\
\hline 6. Educación & 54.53 & -0.57 & 6. Educación & 54.37 & -0.85 \\
\hline
\end{tabular}

Fuente: Cálculos propios basados en la MESEP. 
Tabla A.6. Descomposición de los Cambios en el Coeficiente de Gini: Ingreso Laborales en Colombia, MESEP 2002 - MESEP 2012

\begin{tabular}{|c|c|c|c|c|c|}
\hline \multicolumn{3}{|l|}{ Período base 2002} & \multicolumn{3}{|l|}{ Período base 2012} \\
\hline \multirow{2}{*}{ Indicador } & \multicolumn{2}{|c|}{ Ingresos } & \multirow{2}{*}{ Indicador } & \multicolumn{2}{|c|}{ Ingresos } \\
\hline & Nivel & Cambio & & Nivel & Cambio \\
\hline 2002 observado & 56.56 & & 2002 observado & 56.56 & -3.04 \\
\hline 2012 observado & 53.52 & -3.04 & 2012 observado & 53.52 & \\
\hline Efecto & & & Efecto & & \\
\hline 1. Retornos a la educación & 55.87 & -0.69 & 1. Retornos a la educación & 54.67 & -1.15 \\
\hline 2. Brecha de género & 56.35 & -0.21 & 2. Brecha de género & 54.35 & -0.83 \\
\hline 3. Retornos a la experiencia & 56.51 & -0.05 & 3. Retornos a la experiencia & 54.27 & -0.75 \\
\hline 4. Factores no observados & 55.77 & -0.79 & 4. Factores no observados & 54.81 & -1.29 \\
\hline 5. Empleo & 57.40 & 0.84 & 5. Empleo & 53.05 & 0.47 \\
\hline Horas de trabajo & 56.28 & 0.72 & Horas de trabajo & 53.03 & 0.49 \\
\hline Participación & 56.70 & 0.14 & Participación & 53.55 & -0.03 \\
\hline 6. Educación & 56.04 & -0.52 & 6. Educación & 54.10 & -0.58 \\
\hline
\end{tabular}

Fuente: Cálculos propios basados en la MESEP.

Tabla A.7. Salario horario real por nivel educativo para hombres y mujeres, años seleccionados Salario real (\$2008)

\begin{tabular}{|c|c|c|c|c|c|c|c|c|c|c|}
\hline \multirow{2}{*}{ Nivel educativo } & \multicolumn{2}{|c|}{1992} & \multicolumn{2}{|c|}{1996} & \multicolumn{2}{|c|}{2002} & \multicolumn{2}{|c|}{2005} & \multicolumn{2}{|c|}{2012} \\
\hline & Hombre & Mujer & Hombre & Mujer & Hombre & Mujer & Hombre & Mujer & Hombre & Mujer \\
\hline Primaria incompleta & & 2144.6 & 1.8 & 2349.6 & 1821.1 & 1700.9 & 1808.6 & 1736.3 & 2246.2 & 2021.3 \\
\hline Primaria completa & 2850.6 & 2253.8 & 3.6 & 2569.9 & 2 & 2025.2 & 5.8 & 1947.9 & 11.3 & 2372.6 \\
\hline Secundaria incompleta & 3300.0 & 2736.5 & 3.9 & 3215.3 & 2 & 2586.6 & 2.5 & 2377.7 & 6.5 & 16.2 \\
\hline Secundaria completa & 4398.5 & 5.5 & 5368.5 & 4177.4 & 3487.9 & 3279.4 & 3348.4 & 3072.3 & 3922.7 & 3162.9 \\
\hline Superior incompleta & 6153.7 & 5499.4 & 8316.4 & 6056.9 & 7303.2 & 5956.9 & 5729.9 & 5415.4 & 6033.5 & 5083.0 \\
\hline Sun & 11793.2 & 7970.0 & 15867.0 & 11064.6 & 13279.1 & 11296.1 & 12871.8 & 9868.7 & 15001.4 & 12022.6 \\
\hline Total & 4429.3 & 3690.2 & 5376.2 & 4406.4 & 3645.8 & 3972.3 & 3636.2 & 3765.8 & 4373.0 & 4285.6 \\
\hline
\end{tabular}

Nota: los datos cubren a trabajadores entre 12 y 65 años.

Fuente: Cálculos propios basados en la ENH y MESEP. 
Tabla A.8. Ecuación de selección de la tabla 4.2: Variable dependiente $=1$ si salario horario $>0$

\begin{tabular}{|c|c|c|}
\hline \multicolumn{3}{|c|}{ ENH (urbano) } \\
\hline & 1992 & 1996 \\
\hline \begin{tabular}{|l|} 
Primaria \\
completa
\end{tabular} & $\begin{array}{l}0.0382 \\
(1.42)\end{array}$ & $\begin{array}{l}0.0245 \\
(0.68)\end{array}$ \\
\hline $\begin{array}{l}\text { Secundaria } \\
\text { incompleta }\end{array}$ & $\begin{array}{l}0.0463 \\
(1.87)\end{array}$ & $\begin{array}{c}0.0094 \\
(0.29)\end{array}$ \\
\hline $\begin{array}{l}\text { Secundaria } \\
\text { completa }\end{array}$ & $\begin{array}{c}0.0903^{\star *} \\
(3.16)\end{array}$ & $\begin{array}{c}0.148^{\star \star *} \\
(4.23)\end{array}$ \\
\hline $\begin{array}{l}\text { Superior } \\
\text { incompleta }\end{array}$ & $\begin{array}{c}0.340^{\star \star \star} \\
(8.49)\end{array}$ & $\begin{array}{c}0.409^{\star * *} \\
(8.23)\end{array}$ \\
\hline $\begin{array}{l}\text { Superior } \\
\text { completa }\end{array}$ & $\begin{array}{c}0.109^{* *} \\
(2.79)\end{array}$ & $\begin{array}{c}0.231^{* * *} \\
(4.80)\end{array}$ \\
\hline Hombre & $\begin{array}{l}0.555^{\star * *} \\
(29.69)\end{array}$ & $\begin{array}{c}0.624^{* * *} \\
(30.57)\end{array}$ \\
\hline Edad & $\begin{array}{c}0.0401^{* * *} \\
(8.29)\end{array}$ & $\begin{array}{c}0.0591^{* * *} \\
(11.42)\end{array}$ \\
\hline $\begin{array}{l}\text { Edad al } \\
\text { cuadrado }\end{array}$ & $\begin{array}{c}-0.000811^{* * *} \\
(13.28)\end{array}$ & $\begin{array}{c}-0.00104^{* * *} \\
(15.87)\end{array}$ \\
\hline Casado & $\begin{array}{c}-0.888^{* * *} \\
(40.10)\end{array}$ & $\begin{array}{c}-0.933^{* * *} \\
(38.22)\end{array}$ \\
\hline $\begin{array}{l}\text { Hijos } \\
\text { menores }\end{array}$ & $\begin{array}{c}0.0389^{* * *} \\
(6.82)\end{array}$ & $\begin{array}{c}0.0213^{* *} \\
(3.27)\end{array}$ \\
\hline Menor de 18 & $\begin{array}{c}-0.825^{\star * *} \\
(20.43)\end{array}$ & $\begin{array}{c}-0.765^{\star * *} \\
(16.51)\end{array}$ \\
\hline Asiste & $\begin{array}{c}-0.940^{\star * *} \\
(27.77)\end{array}$ & $\begin{array}{c}-0.936^{\star * *} \\
(23.66)\end{array}$ \\
\hline $\begin{array}{l}\text { Jefe } \\
\text { ocupado }\end{array}$ & $\begin{array}{l}1.611^{* * *} \\
(61.20)\end{array}$ & $\begin{array}{l}1.908^{* * *} \\
(61.36)\end{array}$ \\
\hline $\begin{array}{l}\text { Cónyuge } \\
\text { ocupado }\end{array}$ & $\begin{array}{c}2.167^{\star \star \star} \\
(63.95)\end{array}$ & $\begin{array}{c}2.425^{\star \star *} \\
(59.74)\end{array}$ \\
\hline Constante & $\begin{array}{c}-0.799^{* * *} \\
(9.01)\end{array}$ & $\begin{array}{c}-1.071^{* * *} \\
(11.08)\end{array}$ \\
\hline
\end{tabular}

\begin{tabular}{|c|c|c|c|}
\hline \multicolumn{4}{|c|}{ MESEP } \\
\hline & 2002 & 2005 & 2012 \\
\hline $\begin{array}{l}\text { Primaria } \\
\text { completa }\end{array}$ & $\begin{array}{c}0.0623^{*} \\
(2.44)\end{array}$ & $\begin{array}{c}0.0500^{* *} \\
(2.92)\end{array}$ & $\begin{array}{l}0.0326 \\
(1.95)\end{array}$ \\
\hline $\begin{array}{l}\text { Secundaria } \\
\text { incompleta }\end{array}$ & $\begin{array}{l}0.0383 \\
(1.57)\end{array}$ & $\begin{array}{c}0.0186 \\
(1.18)\end{array}$ & $\begin{array}{c}0.0666^{\star \star \star} \\
(4.39)\end{array}$ \\
\hline $\begin{array}{l}\text { Secundaria } \\
\text { completa }\end{array}$ & $\begin{array}{c}0.156^{* * *} \\
(6.27)\end{array}$ & $\begin{array}{c}0.156^{\star \star *} \\
(9.28)\end{array}$ & $\begin{array}{c}0.180^{\star * \star} \\
(11.79)\end{array}$ \\
\hline $\begin{array}{l}\text { Superior } \\
\text { incompleta }\end{array}$ & $\begin{array}{c}0.347^{* * *} \\
(10.87)\end{array}$ & $\begin{array}{c}0.347^{* * *} \\
(16.28)\end{array}$ & $\begin{array}{c}0.422^{* * *} \\
(23.98)\end{array}$ \\
\hline $\begin{array}{l}\text { Superior } \\
\text { completa }\end{array}$ & $\begin{array}{c}0.613^{* * *} \\
(19.62)\end{array}$ & $\begin{array}{l}0.555^{\star * *} \\
(24.33)\end{array}$ & $\begin{array}{c}0.642^{* \star *} \\
(31.51)\end{array}$ \\
\hline Hombre & $\begin{array}{c}0.707^{\star * *} \\
(50.14)\end{array}$ & $\begin{array}{c}0.800^{\star * \star} \\
(79.78)\end{array}$ & $\begin{array}{c}0.770^{\star \star \star} \\
(86.81)\end{array}$ \\
\hline Edad & $\begin{array}{c}0.0985^{* * *} \\
(26.25)\end{array}$ & $\begin{array}{c}0.115^{\star * *} \\
(44.37)\end{array}$ & $\begin{array}{c}0.111^{* * *} \\
(49.38)\end{array}$ \\
\hline $\begin{array}{l}\text { Edad al } \\
\text { cuadrado }\end{array}$ & $\begin{array}{c}-0.00147^{\star * *} \\
(31.67)\end{array}$ & $\begin{array}{c}-0.00170^{\star \star *} \\
(50.96)\end{array}$ & $\begin{array}{c}-0.00157^{* * *} \\
(55.61)\end{array}$ \\
\hline Urbano & $\begin{array}{c}-0.0036 \\
(0.17)\end{array}$ & $\begin{array}{c}-0.0064 \\
(0.47)\end{array}$ & $\begin{array}{c}0.0653^{* * *} \\
(5.35)\end{array}$ \\
\hline reg02 & $\begin{array}{l}0.0340 \\
(0.60)\end{array}$ & $\begin{array}{c}0.0566 \\
(1.25)\end{array}$ & $\begin{array}{c}0.557^{\star \star *} \\
(18.07)\end{array}$ \\
\hline Región 03 & $\begin{array}{l}0.0282 \\
(0.50)\end{array}$ & $\begin{array}{c}0.0234 \\
(0.53)\end{array}$ & $\begin{array}{c}0.392^{* * *} \\
(13.56)\end{array}$ \\
\hline Región 04 & $\begin{array}{c}0.0068 \\
(0.12)\end{array}$ & $\begin{array}{c}-0.0403 \\
(0.91)\end{array}$ & $\begin{array}{c}0.352^{* * *} \\
(12.20)\end{array}$ \\
\hline Región 05 & $\begin{array}{c}0.0172 \\
(0.28)\end{array}$ & $\begin{array}{c}0.0543 \\
(1.19)\end{array}$ & $\begin{array}{c}0.309^{\star \star \star} \\
(10.46)\end{array}$ \\
\hline Casado & $\begin{array}{c}-1.106^{\star * \star} \\
(62.14)\end{array}$ & $\begin{array}{c}-1.083^{* * *} \\
(88.15)\end{array}$ & $\begin{array}{c}-0.993^{\star * *} \\
(55.86)\end{array}$ \\
\hline $\begin{array}{l}\text { Hijos } \\
\text { menores }\end{array}$ & $\begin{array}{c}0.0382^{* * *} \\
(7.54)\end{array}$ & $\begin{array}{c}0.0418^{* * *} \\
(12.84)\end{array}$ & $\begin{array}{c}0.0337^{* * *} \\
(10.45)\end{array}$ \\
\hline Menor de 18 & $\begin{array}{c}-0.523^{\star \star *} \\
(17.08)\end{array}$ & $\begin{array}{c}-0.538^{\star * *} \\
(24.74)\end{array}$ & $\begin{array}{c}-0.676^{* * *} \\
(34.88)\end{array}$ \\
\hline Asiste & $\begin{array}{c}-0.808^{\star \star *} \\
(30.83)\end{array}$ & $\begin{array}{c}-0.882^{\star * *} \\
(48.98)\end{array}$ & $\begin{array}{c}-0.636^{* * *} \\
(45.34)\end{array}$ \\
\hline Jefe ocupado & $\begin{array}{c}2.968^{\star * *} \\
(75.67)\end{array}$ & $\begin{array}{l}3.036^{\star * *} \\
(110.17)\end{array}$ & $\begin{array}{l}2.832^{\star * \star} \\
(134.00)\end{array}$ \\
\hline $\begin{array}{l}\text { Cónyuge } \\
\text { ocupado }\end{array}$ & $\begin{array}{c}2.485^{\star \star *} \\
(96.72)\end{array}$ & $\begin{array}{l}2.455^{* * *} \\
(133.22)\end{array}$ & $\begin{array}{l}2.366^{\star \star \star} \\
(155.66)\end{array}$ \\
\hline Constante & $\begin{array}{c}-1.954^{* * *} \\
(22.99)\end{array}$ & $\begin{array}{c}-2.167^{* * *} \\
(34.09)\end{array}$ & $\begin{array}{c}-2.583^{* * *} \\
(51.13)\end{array}$ \\
\hline
\end{tabular}

Nota: los datos representan estimación tipo Heckman por máxima verosimilitud; los valores t están entre paréntesis. Los datos cubren a los individuos entre 12 y 65 años de edad. ${ }^{*} p<0.05{ }^{* *} p<0.01{ }^{* * *}$ $p<0.001$

Fuente: Cálculos propios basados en la ENH-Urbana y MESEP 